\title{
Die Theorie der Herstellung und Stabilität kolloider Lösungen und Niederschläge. II.
}

Von P. P. von We i marn (St. Petersburg). $\begin{gathered}\text { (Eingegangen } \\ \text { ain 12. Okt. fol1) }\end{gathered}$ (Meinem Freunde W'olfgang Ostwald gewidmet.)

b) Die wichtigsten Grundlagen der allgemeinen Theorie der Kondensations-Methoden zur Herstellung disperser Systeme. ')

\section{Einleitung.}

In meinen früheren Abhandlungen*) habe ich hăufig die Bedingungen zur Herstellung von dispersen Systemen - kolloiden Lösungen, Suspensionen und kolloiden Niederschlägen - mit Hilfe der Kondensation behandelt; destralb führe ich in dieser Ahhandlung vergleichsweise nur ganz kurz die Hauptresultate meiner theoretischen und experimentellen Untersuchungen der kolloiden Kondensationserscheinunget an, wobei die Ausfïhrungen derartig abgefaBt sein werden, daB erstens der mit meinen früheren Abhandlungen unbekannte Leser keine Schwierigkeiten beim Verstehen empfinden wird, und zweitens, daB er die charakteristischsten die Kondensationstheorie erklärenden Erscheinungen mit Hilfe von äuBerst einfachen Experimenten in seinem eigenen Laboratorium wiederholen kann. Eine besonilere Aurinerksamkeit wird der Auslegung der Gründe für die Stabilität hochdisperser Systeme -- kolloider Losungen, Suspensionen und kolloider Niederschlăge - geschenkt, denn diese wichtige Frage ist, meiner Meinung nach, noch nicht genügend beleuchtet worden und deshalb auch noch nicht vollständig verständlich.

1) Der erste Tell dieser Abhandlung a) ,Dle wichtigsten Grundlagen der allgemeinen Theorie der Dispersionsmethoden $z$ ur Herstellung disperser System $e^{*}$ ist in d. Kolloidchem. Beih. 1, 396-422 (1910) abgedruckt.

2) Koll.-Zeitschr. 2-8 (1907-1911) und besonders "Grundz üge d. Dispersoidchemie“ (Dresden 1911). 


\section{Die Herstellung von dispersen Nlederschlăgen.}

A. Versuche. Wenn wir eine 2. B. bei $70^{\circ} \mathrm{C}$ gesâttigte $\mathrm{NaCl}-$ Lösung langsam bis zur Zimmertemperatur abkühlen, so erhalten wir nit unbewaffnetem Auge deutlich sichtbare Kristalle; wiederholen wir denselben Versuch jedoch bei schneller Abkühlung bis zur Zimmertemperatur, so fallen die Kristalle viel kleiner aus; tauchen wir endlich eine alkoholische NaCl-Lösung in einem zugeschmolzenen Probiergläschen schnell in flüssige Luft, so scheidet sich das Natriumchlorid 1) in so hochdispersem Zustande aus, daB es unmöglich ist, die einzelnen Kristallchen zur unterscheiden ... wir erhalten ein durchsichtiges Glas (P. P. von Weimarn und J. B. Kahan). Führen wir jetzt mit einer bei Zimmertemperatur gesättigten wässerigen $\mathrm{NaCl}$-Lösung und Propylalkohol folgende Versuche aus: GieBen wir in ein Probiergläschen je $5 \mathrm{ccm}$ Propylalkohol und Na Cl-Lösung (es entstehen zwei Schichten) und schütteln die Flüssigkeit durch, so erhalten wir einen dispersen $\mathrm{NaCl}$-Niederschlag; bei einer 300 fachen Vergrößerung (Objektiv $4 \mathrm{~b}$, Okular 18) erscheinen die Niederschlagskörnchen als Würfel mit Kanten von $2-3 \mathbf{m m}$, von welchen einige noch nicht ganz ausgebildet und die kleinsten Würfelchen zu Flocken vereinigt sind [Ausflockung nach G. Malfitano $\left.{ }^{2}\right)$ ]. Nehmen wir auf $5 \mathrm{~cm} \mathrm{NaCl}$ Lösung $20 \mathrm{ccm}$ Propylalkohol (es entstehen zwei Schichten), so erhalten wir einen noch höher dispersen Niederschlag, dessen Körnchen bei einer 300 fachen Vergrößlerung Würfel und Kanten von $0,5-1 \mathrm{~mm}$ darstellen. Die Bildung. von Flocken ist in diesem Fall noch deutlicher (die Ausflockung erfolgt intensiver). Schüttelt man aber $2 \mathrm{ccm}$ der $\mathrm{NaCl}-\mathrm{Lösung}$ mit $40 \cdots 50 \mathrm{ccm}$ Propylalkohol (hierbei wird alles Wasser durch den Alkohol extrahiert), so erhält man einen flockigen $\mathrm{NaCl}$-Niederschlag, der bei einer 300 fachen VergröBerung "amorph" erscheint; er besteht aus Flocken von körniger Struktur und bei der angeführten Vergrößerung kann man keine Würfelform der Körnchen feststellen (die Ausflockung erhält eine deutliche Struktur). Im Laufe der Zeit bilden die Körnchen (Umkristallisation siehe weiter) infolge der Zunahme ihres Volums kleinste Würfelchen ${ }^{3}$ ).

1) Genauet zu sagen das Natriumchloridsolvat.

8) G. Malfitano, Kolloidchem. Belh. 2, 142- 212 (1910).

3) Die Lóslichkeit von $\mathrm{NaCl}$ in einer Mischung von Propylalkohot und Wasser ist noch nicht bis zum genligenden Minimum gebracht worden.

4) AuBerdem erfordert ithe Austührung eine bedeutende Routine in der Herstellung von mikroskopischen Präparaten, weil der Propylalkohol schnell ver- 
v. Weim arn, Die Theorie der Herstellung und Stabilităt kolloider Lösungen usw.

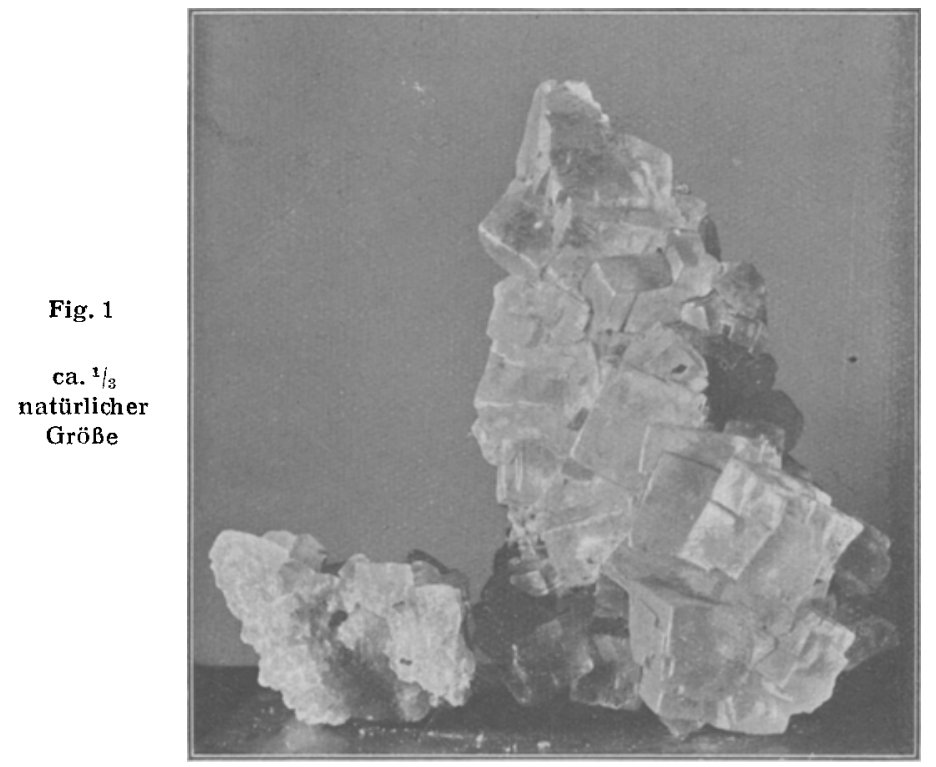

TAFEL I

Fig. 2

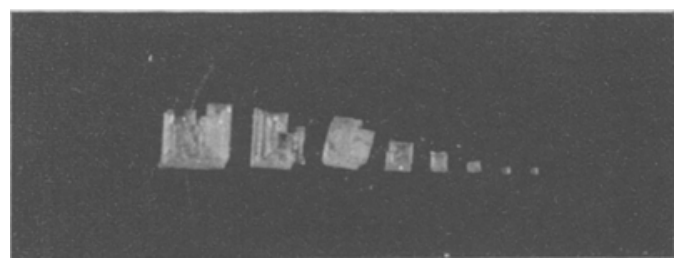

Fig. 3

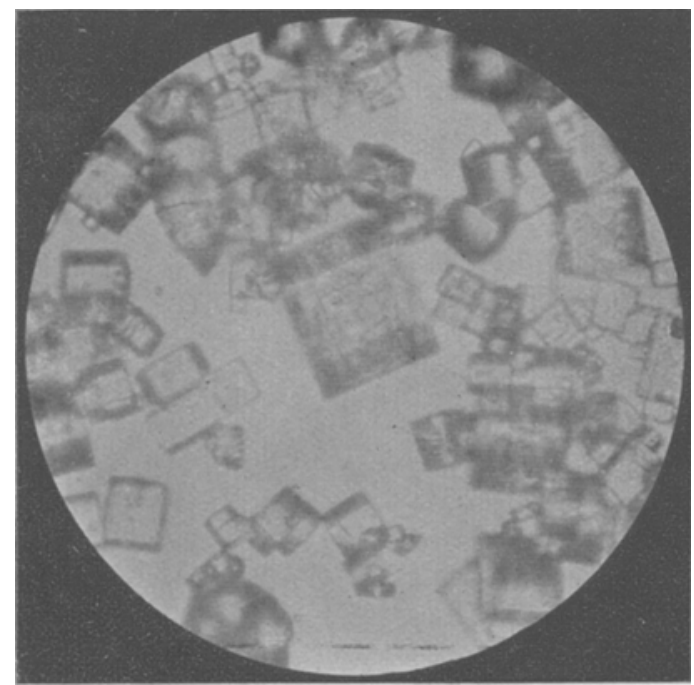


Die eben angeführten Versuche init Propylalkohol und gesättigter wässeriger $\mathrm{NaCl}$-Lösung sind allerdings weniger belehrend ${ }^{3}$ ) als die, welcher ich mich bei den Demonstrationen in den Vorlesungen über Kolloidchenie bediene. Diese letzteren werden durch die Tafeln I, II und III illustriert und durch folgende Erklärungen verständlich: Auf der Tafel I stellt Fig. 1 zwei NaCl-Kristalldrusen dar, welche sich in der Natur bei langsamem Entstehen der festen Phase aus ungeheuren Losungsquanten entwickelt hahen; die ersten fïnf Kristalle in Fig. 2 der Tafel I stammen ebenfalls aus der Natur (sogenanntes angeschossenes Salz), die übrigen drei wurden durch Kristallisation aus wässerigen $\mathrm{NaCl}-\mathrm{L}$.ßsungen bei gewöhnlichen Bedingungen im Laboratorium erhalten. Alle Kristalle in der Tafel I sind in ca. Is natürlicher Größe abgebildet. Fig. $3 ; z u 15 \mathrm{ccm}$ einer gesättigten wasserigen $\mathrm{NaCl}$-Lösung wurden $20 \mathrm{ccm}$ Methylalkohol zugesetzt, der Niederschlag 15 Minuten später auf ein Objektivglas gebracht, mit einem Deckgläschen bedeckt und photographiert. Wie man aus dem Mikrogramm ersieht, besteht der Niederschlag aus Würfeln. (Objektiv 4b, Okular 18, Vergroberung ca. 300 fach.) Die NaCl-Niederschlage der Tafel II und III wurden auf folgende Weise hergestellt: Fig. 4; zu $40 \mathrm{~cm}$ Propylalkohol wurden $4 \mathrm{ccm}$ einer gesättigten Lossung von $\mathrm{NaCl}$ in Methylalkohol hinzugefügt und der Niederschlag nach 30 Minuten photographiert (unter denselben Bedingungen wie beim vorhergehenden Versuch). Der Niederschlag stellt ein Gemenge von Würfeln (in geringer Anzahl) und Würfel-Oktaeder dar; die Oktaederflăchen sind hăufig nicht ganz regelmäRig entwickelt ${ }^{1}$ ). Infolge von lichtreflexen sind die Würfel-Oktaeder auf Fig. 4 nicht relief. Fig. $5 ; 4 \mathrm{ccm}$ einer gesättigten Lösung von $\mathrm{NaCl}$ in Methylalkohol wurden in eine Mischung von $30 \mathrm{ccm}$ Propylalkohol und $10 \mathrm{ccm}$ Aether gegossen und nach 1 Stunde 20 Minuten der Niederschlag photographiert (die Herstellung des Präparates und die Vergrößerung wie in Pig. 3 und Fig. 4). Der Niederschlag stellt Wachstumstiguren dar ${ }^{2}$ ). Fig. $6 ; 4 \mathrm{~cm}$ einer gesăttigten Lösung von $\mathrm{NaCl}$ in Metlyylalkohol wurden in eine Mischung von $20 \mathrm{ccm}$ Propylalkohol und $20 \mathrm{ccm}$ Aether gegossen. Nach 3 Stunden 30 Minuten ist die l.ösung

dunstet und die Loslichkeit des Niederschlages zunimmt, weshalb die Kornchen sich schneller umkristallisieren.

1) Die kristallographische Untersuchung dieses Niederschlages wird im Berginstitut ausgeführt.

7) Die Oberflache der Wachstumsfiguren ist grober als die der holuedrischen Kristalle bel gleichem Volumen. 
noch nicht ganz klar geworden, der größte Teil des Niederschlages hat sich jedoch am Boden des Probiergläschens gesammelt, und der letztere wurde bei einer $6 \mathrm{mal}$ größeren VergröBerung als bei Fig. 3-5 photographiert (Objektiv $3 \mathrm{~mm}$, Okular 18, VergröBerung 1800), weil bei einer 300 fachen Vergrößerung derselbe "amorph" feinkörnig erscheint. Wie aus Fig. 6 zu ersehen ist, stellen die gröBeren Körnchen Kristallskelette dar. Fig. 7; in eine Mischung von $30 \mathrm{ccm}$ Aether und $10 \mathrm{ccm}$ Propylalkohol wurden $4 \mathrm{ccm}$ einer gesăttigten Lösung von $\mathrm{NaCl}$ in Methylalkohol gegossen. Die milchigtrübe Flüssigkeit, die nach 24 Stunden noch nicht klar geworden ist, gibt einen voluminösen Niederschlag von feinkörniger "amorpher" Struktur. Fig. 8; in $40 \mathrm{ccm}$ Aether wurden $4 \mathrm{ccm}$ einer gesättigten Lösung von $\mathrm{NaCl}$ in Methylalkohol gegossen; die stark opaleszierende Flüssigkeit von für dispersoide Systeme charakteristischer rötlichbrauner Färbung im durchfallenden Lichte ergibt nach 24 Stunden nur einen minimalen Niederschlag [Fig. $81^{1}$ ), die dispersoide Lősung ist aber im übrigen unveråndert.

GieBt man weniger als $4 \mathrm{ccm}$ einer gesättigten Lösung von $\mathrm{NaCl}$ in Methylalkohol in stark gekühlten Aether (z. B. - 80 ${ }^{\circ}$ ), so erhalten wir dispersoide Lösungen bis fast zum molekularen Dispersitätsgrade. Man muf in Betracht ziehen, daß die Löslichkeit der festen $\mathrm{NaCl}$ Phase in den erwähnten Mischungen progressiv abnimmt, die Geschwindigkeit ihrer Bildung aber wăchst. Die hier beobachteten Beziehungen sind analog den von mir in früheren Abhandlungen geschilderten von $\mathrm{BaSO}_{4}$. Ergänzend zu den Photogrammen der $\mathrm{BaSO}_{4}$-Niederschläge füge ich hier solche von natürlichen Bariumsulfat-Kristallen (Tafel III, Fig. 9) hinzu.

Nicht nur $\mathrm{NaCl}$, sondern alle Salze der Alkalimetalle $(\mathrm{Na}, \mathrm{K}, \mathrm{Li}$, $\mathrm{Rb}, \mathrm{Cs}, \mathrm{NH}_{4}$ ) des Typs: $\mathrm{RCl}, \mathrm{RBr}, \mathrm{RJ}, \mathrm{RCN}, \mathrm{RCNS}, \mathrm{R}_{2} \mathrm{CO}_{3}$, $\mathrm{R}_{2} \mathrm{SO}_{4}, \mathrm{RNO}_{3}$ kann man auf demselben Wege in verschiedenen Dispersitatsgraden erhalten (P. P. von Weimarn und A. L. Stein), und es liegt kein Grund vor, an der allgemeinen Anwendbarkeit der angeführten Methode (cf. meine früheren Abhandlungen) auf einen beliebigen Körper $z u z w e i f e l n$.

Lösen wir im Amylalkohol so viel metallisches Natrium, um eine bei Zimmertemperatur fast gesättigte Lösung von Natriumamylat in Amylalkohol zu crhalten, und leiten durch dieselbe (bei Abkühlung

1) Es muß bemerkt werden, daß bei diesen Niederschlägen (Pig. 7 und Fig. 8 ; leicht sckundare Strukturen, Wabenstrukturen" und ähnliche, entstehen. 
v. Weimarn, Die Theorie der Herstellung und Stabilität kolloider Lösungen usw.

TAFEL II

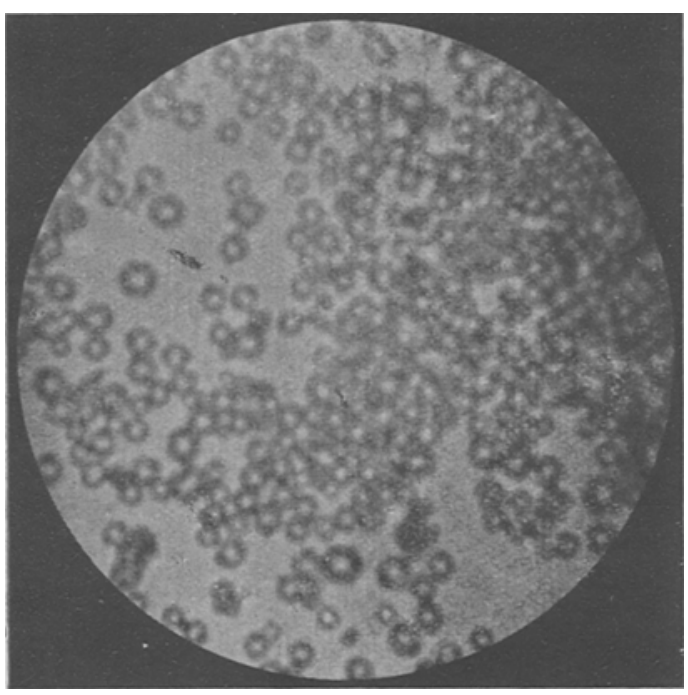

Fig. 4

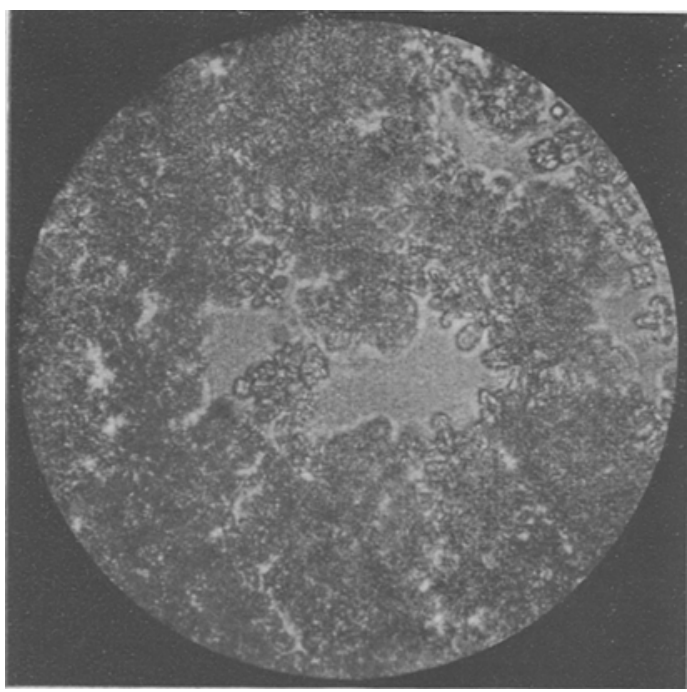

Fig, 6

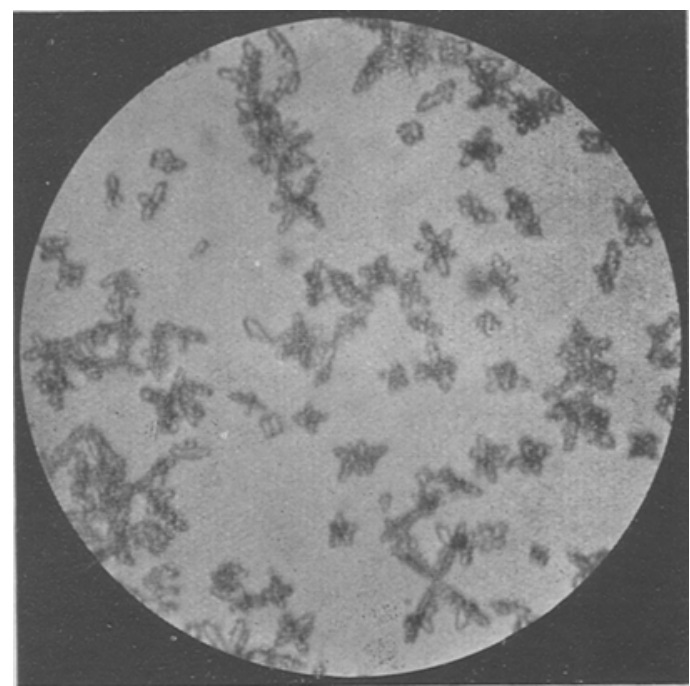

Fig. 5

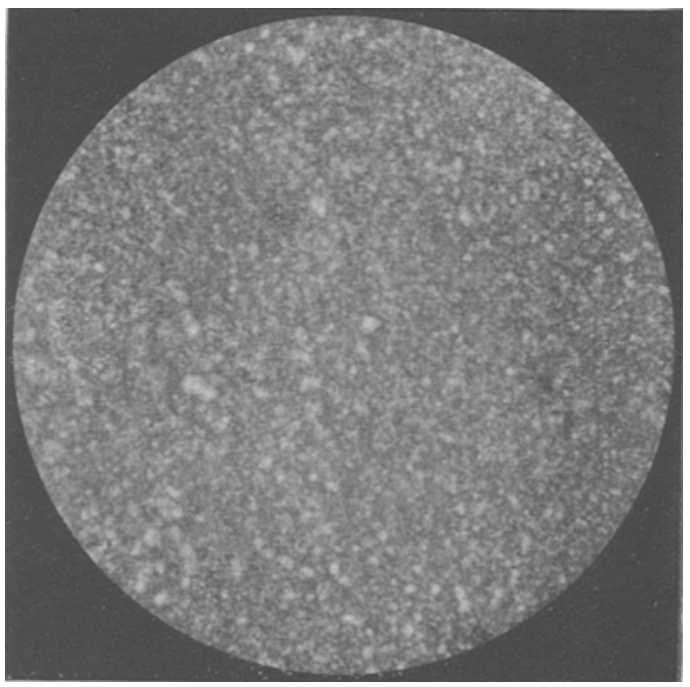

Fig. 7 
durch Eis und Kochsalz) trockenes $\mathrm{HCl}$, so entsteht eine weiblich trübe etwas durchscheinende $\mathrm{NaCl}$-Gallerte (nach der Analyse der gut gewaschenen Gallerte enthält sie ca. 98 Proz. $\mathrm{NaCl}$ ). Wiederholen wir denselben Versuch mit einer Lösung von Natriumamylat in Amylalkohol, welche im Liter 1/100 Gramm-Molekül metallisches Natrium enthălt, so erhalten wir eine stark opaleszierende, weiBlich trübe Flüssigkeit, welche auch nach einer Woche nicht ganz durchscheinend wird (P. P. von Weimarn und J. B. Kahan). In diesem Fall haben wir es mit einer feinen $\mathrm{NaCl}$-Suspension zu tun. ${ }^{1}$ )

Die geschilderten Experimente kann man mil demselben Erfolge mit einem beliebigen Körper ausführen, z. B. mit Natriumphosphat und -azetat u.a., und sie sind tatsăchlich in meinem Laboratorium an den verschiedensten Substanzen ${ }^{2}$ ) mit genau den gleichen Resultaten erprobt worden.

B. Die Theorie der Erscheinungen. Aus den angeführten Versuchen, die der Leser ohne besondere Schwierigkeiten im Laboratorium in Probiergläschen kontrollieren kann, ergeben sich zwei SchluBfolgerungen: 1. mit zunehmender Ausscheidungs geschwindigkeit des Niederschlages vergrobert sich auch schnell die Dispersitat, 2. bei hochdispersen kristallinischen Niederschlagen nimmt mit zunehmendem Dispersitatsgrade die Tendenz zurFlockenbildung $z u$. Die letztere Erscheinung wollen wir als "Ausflockung" bezeichnen (G. Malfita no, 1. c.).

Beim Erhalten des dispersen $\mathrm{NaCl}$ in Wasser und Alkohol durch Abkühlung wird die Niederschlagsbildung dank dem schnellen Uebergange von der Temperatur, wo die Löslichkeit der grobdispersen Kristalle $L_{1}$ ist, zur Temperatur, wo die Löslichkeit ( $L_{2}$ ) geringer ist, beschleunigt; die Niederschlagsmenge, weiche bei der niedrigsten Temperatur in Form von grobdispersen Kristallen sich ausscheiden könnte, wäre $L_{1}-L_{2}$, wenn $\Delta t$ die Abkühlungszeit (resp. die Ausscheidungszeit) bereichnet, so wird $W=\frac{L_{1}-L_{2}}{A t}$ die Geschwindigkeit der Niederschlagsbildung ${ }^{3}$ ) sein und je größer dieselbe ist, um so größer die Dispersităt des Niederschlages, wie die Versuche uns gezeigt haben.

1) Beim EingieBen einer alkoholischen Losung in Aether (s. oben) erhält man suspensoides $\mathrm{NaCl}$ (P. P.von Weimarn und A. L. Stein), vgl. .Orundzüge" 119--120.

2) Ibidem 119-120 und Koll.-Zeitschr. 3, 89-91 (1908).

9) Hierbei lasse ich wissentlicb die Zunahme der Löslichkeit infolge đer Zunahme des Dispersitätsgrades außer acht (vgl. weiter). 
Bei der Herstellung des dispersen $\mathrm{NaCl}$-Niederschlages mit Hilfe von Propylalkohol erfolgt eine schnelle Ausscheidung des Niederschlages infolge der Wasserentziehung durch den Alkohol aus der gesättigten wässerigen Lösung (welche auch etwas Alkohol enthält); wird nicht genügend Alkohol genommen, um alles Wasser zu entziehen (erster und zweiter Versuch), so erhalt man zwei Fhiissigkeitsschichten und den Niederschlag, ist aber Alkohol genügend vorhanden (dritter Versuch), so erhalt man nur eine flassige Schicht und den Niederschlag!). Die Entziehung des Wassers durch den Alkohol erfolgt aus den Tröpfchen einer wässerigen Lösung, welche im Propylalkohol (genauer in einer Wasserlösung in Propylalkohol) emulgiert sind; es ist verständlich, dat die Niederschlagsbildung un so schneller erfolgt, je kleiner die Tröpfchen ${ }^{2}$ ) sind und je mehr Akohol und weniger der gesättigten wässerigen $\mathrm{NaCl}$-Lösung genommen wird. Bezeichnen. wir mit P die Niederschlagsmenge, welche sich aus einem bestimmten Volumen der Mischung ausscheidet, mit $\boldsymbol{A} t$ die zu dieser Ausscheidung notwendige Zeit, so drïckt

$$
\mathrm{W}=\mathrm{P}
$$

die Geschwindigkeit der Niederschlagsbildung aus, und je grober dieselbe ist, un so grober wird, wie die angeführten Versuche zeigen, die Dispersität des Niederschlages sein. Die Geschwindigkeit können wir im gegebenen Fall bis zum möglichen Maximum steigern, dadurch daB wir (bei energischem Durchschütteln des Alkohols) ein Tropfchen einer $\mathrm{NaCl}-\mathrm{loossung}$ in ein großes Quantum Propylalkohol hineingeben, hierbei wird $\Delta \mathrm{t}$ prakt isch unermeblich klein, und dementsprechend erhallt $W$ einen so großen Wert, daB es für unsere Sinnesorgane unfaklich gruß erscheint. Für die typische kolloide Kondensation sind, wie man aus den Versuchen mit $\mathrm{NaCl}$ in Methyl-, Propyl-, Amylalkohol und Aether ersieht, noch bedeutendero Geschwindigkeiten der Niederschlagsbildung erforderlich, weil $z$. B. bei der Entstehung der $\mathrm{NaCl}$-Gallerte das Chlornatrium fast "momentan" sich ausscheidet. Aus allem Angeführten geht klar hervor, daki man, un sich im Gebiet solcher sich schwer vorzustellenden Geschwindigkeiten zu orientieren, einen Faktor ausfindig machen muB, der uns die Möglichkeit geben würde, diese Geschwindigkeiten, natürlich relativ genom in en,

1) Aehniiche Erschesnungen der Schichtenbildung zeichnen sich durcl groBe Allgemeinheit aus und liegen der allgemeinen Herstellungsmethode der Enulsoide 2u Grunde, cf. „Grundzuge" 106-107, 109.

2) Koll.-Zeitsclir. 2, 230-231 (1908). 
v. Weimarn, Die Theorie der Herstellung und Stabilität kolloider Lösungen usw.

TAFEL III

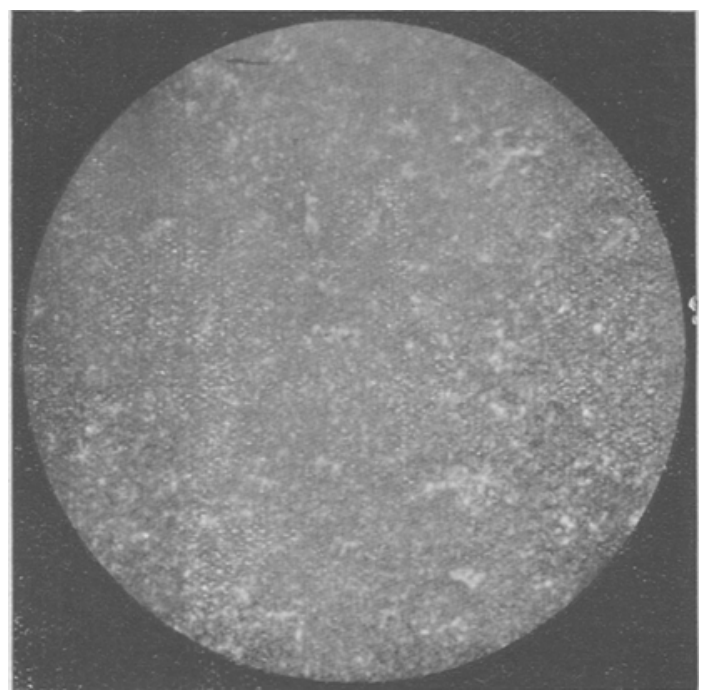

Fig. 8

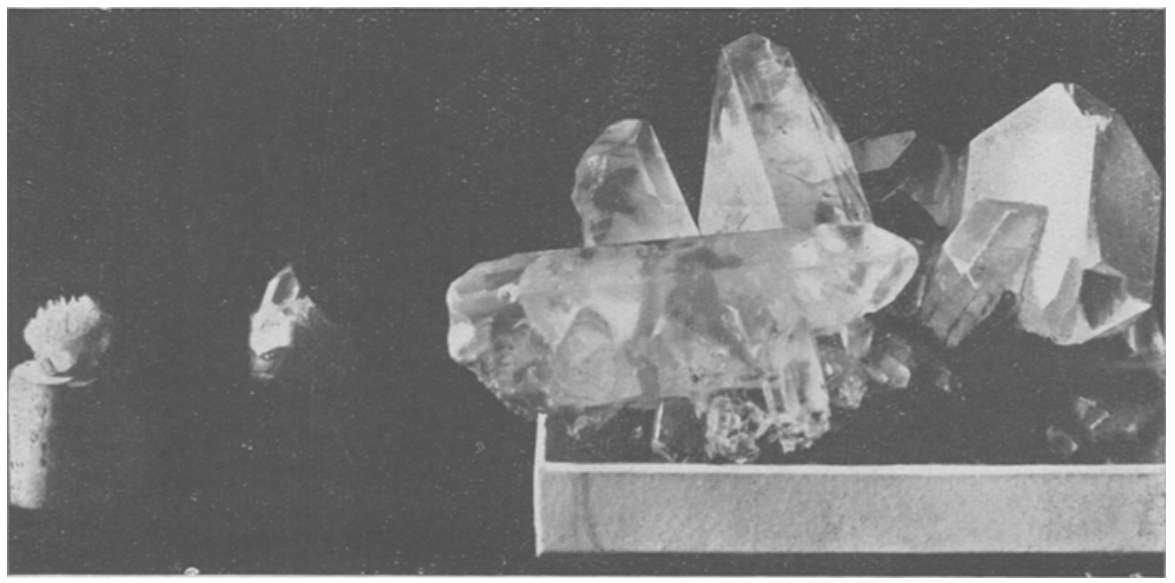

Natürliche Bariumsulfatkristalle

Fig. 9

ca. ${ }^{1}$, natürlicher Größe 
abzuschătzen. Dieser Faktor ist von mir schon im Jahre 1906 gefunden und "Nieder sch lag s formk oeffizient" genant worden"); spăter $^{2}$ ) wurde er bei der Verallgemeinerung auch auf die übersättigten Losungen in "anfänglicher Uebersätigungsgrad" umbenannt; man kann diesen Faktor auch als „Dispersitätsgradkoeffizient des dispersen Systems" bezeichnen, weil mit dieser Bezeichnung noch nicht ausgedrückt ist, ob der Niederschlag schon ausgefallen ist oder nicht $(\boldsymbol{z}$. B. die Blldung einer kolloiden Losung). Den Dispersitătsgradkoeffizient wollen wir fernerhin durch die Formel

$$
\mathrm{U}=\frac{\mathrm{Q}-\mathrm{L}}{\mathrm{L}}=\frac{\mathrm{P}}{\mathrm{I}}
$$

bezeichnen, worin $Q-L$ die Menge des Stoffes ausdrückt, welche nach langerer oder kürzerer Zeit als grobdisperse Phase sich ausscheiden muB, $L$ die Löslichkeit der grobdispersen Phase ${ }^{3}$ ). Weshalb ich es für zweckmabig gehalten habe in die Formel die obigen Werte zu bringen und nicht andere, wird sich aus den weiteren Ausfährungen ergeben. Aus den experimentellen Daten werden wir sofort sehen, auf welche Weise $U$ die relativen Geschwindigkeiten der Ausscheidung der dispersen Phase abzuschätzen gestattet. Führen wir die Reaktion zur Herstellung z. B. von Ba SO$O_{4}\left[\mathrm{Ba}(\mathrm{CNS})_{2}+\mathrm{CoSO}_{4}=\mathrm{BaSO}_{4}+\mathrm{Co}(\mathrm{CNS})_{2}\right]$ in Wasser und in 50 Proz. Alkohol (in beiden Dispersionsmitteln verlăuft die Reaktion für unsere Sinnesorgane momentan), und nehmen wir an, daB in beiden Fällen die gleiche Substanzmenge sich ausscheidet, z. B. $0,5 \mathrm{~g}$, so sehen wir, dab wir im Wasser einen! weniger dispersen (mikrokristallinischen) Niederschlag erhalten, als im wässerigen Alkohol, in dem ein gallertartiger Niederschlag entsteht. Aus den schon angeführten Versuchen mit mebbaren Geschwindigkeiten (Abkühlung von wässerigen $\mathrm{NaCl}$-Lösungen usw.) wissen wir, daß der Dispersitătsgrad um so größer ist, je schneller der Niederschlag sich ausscheidet; deshalb ist auch die Schlußfolgerung natürlich, daB die Ausscheidungsgeschwindigkeit von $\mathrm{BaSO}_{4}$ in wässerigem Alkohol größer ist als in Wasser. Zugleich wissen wir auch, daB

1) Vgl. meine Abhandlungen in den bis jetzt erschienenen Banden der Koll.-Zeltschr. und Kolloidchemischen Belhefte.

2) Vgl. Siegfried Burgstaller, Berlin: ,Formales und Genetisches tiber die Bildung fester Losungen bei der Dissoziation von Oxyden*. Abhandl. d. deutsch. naturwiss.-medlz. Vereines fur Bohmen ,Lotos* in Prag 3, 244--251 (Prag 1912). (Bemerk. b. d. Korrektur.)

9) Vgl. "Grundzüge“. 
die Löslichkcit des grobdispersen $\mathrm{BaSO}_{4}$ bedeutend geringer ist im wässerigen Alkohol als im Wasser. Hieraus habe ich gefolgert, dab dic Geschwindigkeit der Bildung der dispersen Phase bei sonst annahernd gleichen Bedingungen un so grofer ist, je kleiner L. Die Tatsache der Zunahme der Dispersitä mit abnehmender Löslichkeit bei sonst gleichen Badingungen ist von mir an Hunderten von Beispielen konstatiert ${ }^{1}$ ). Der Versuch hat weiter gezeigt, dah, wenn man bei fast konstantem $L$ die Konzentration der reagierenden Lösungen vergröBert [z. B. bei Führung der Reaktion in Wasser: $\mathrm{Ba}(\mathrm{CNS})_{2}+\mathrm{MnSO}_{4}=\mathrm{BaSO}_{4}+\mathrm{Mn}(\mathrm{CNS})_{2}$, der Dispersitătsgrad der dispersen Phase wächst, woraus ich folgerie, daB die Geschwindigkeit jhrer Entstehung mit $P z u$ nimmt bei sonst gleichen Bedirgungen, obgleich die Niederschlagsbildung bei äußerst verschiedenen Konzentrationen uns gleich "momentan" erscheint. Ferner hat der Versuch gezeigt, dab nur bei $\frac{P}{L}$ nahen Werten (für einen und denselben Körper in verschiedenen Dispersionsmitteln oder für verschiedene Körper in einem oder verschiedenen Dispersionsmitteln) man mehr oder weniger nahe Resultate inı Sinne dẹs Dispersitätsgrades erhălt. Wenn z. B. bei $\mathrm{P}=1 \mathrm{~g}$ und $\mathrm{L}=10^{-4} \mathrm{~g}$ deutliche Mikrokristalle irgend eines Körpers entstehen, șo muß man für denselben Körper bei $L=10^{-5}$ die Bildung von ähnlichen Mikrokristallen bei $P=0,1 \mathrm{~g}$ erwarten (natürlich nach längerem Zeitintervall), dann wird $\frac{\mathrm{P}}{\mathrm{L}}$ in beiden Pallen gleich (scheinbare Ausnahmen siehe weiter).

Die molekularkinetische Analyse des Kondensationsprozesses ${ }^{2}$ ) führt zu demselben Ergebnis, nämlich, daß die Geschwindigkeit der Entstehung der dispersen Phase in direkter Abhängigkeit vom Wert $U$ sich befindet. Auf Grund dieses Zusummenhanges zwischen der Entstehungsgeschwindigkeit der dispersen Phase und $U$ werden wir zur Analyse des Kondensationsprozesses uns nur des Ausdrucks far $U$ bedienen, weil die absoluten Geschwindigkeitswerte nicht berechnet und von uns nicht unterschieden werden können. Deshalb erscheint auch die Frage nach dem Vorteil bei der Anwendung des Koeffizienten $\mathrm{U}$ durchaus berechtigt. In dicsem Koeffizient sind die Bedingungen

2) Vgl. z. B. die Tabelle in Koll.-Zeitschr. 4, 35, 133 (1909) und .Grundzüge* $119-120$ (1911). (1910).

7) Vgl. z. B. ,Grundzüge* $38-46$ (1911) oder Koll.-Źeitschr. 6, 210-213 
für die Entstehung der dispersen Systeme gedrängt ausgedrückt. Der für $U$ angegebene Ausdruck ist im Vergleich $\mathrm{zu}$ den tatsăchlichen Beziebungen bedeutend verkürzt, denn nicht nur $P$ und $L$ beeinflussen die Kondensation. Ohne auf die Details ${ }^{1}$ ) näher einzugehen, will ich das Beispiel der Abkühlung einer alkoholischen $\mathrm{NaCl}$-Lösung in flüssiger Luft analysieren. In diesem Bcispiel spielt eine hervorragende Rolle ${ }^{2}$ ) die schnelle Viskositätszunahme des Dispersionsnittels (Alkohol), das sich in Glas umwandelt. Es ist verständlich, daA bei einer derartigen Viskosităt eine Vereinigung von hochdispersen Teilchen zu groBeren fast vollständig gehemmt ist. Wenn die in ihrer Wirkung auf die Kondensation dem Wert $\frac{P}{L}$ äquivalenten Viskositätswerte durch eine Reihe von Versuchen festgestellt wären, so mübten wir, indem wir diese Viskositätswerte mit $Z$ bezeichnen, den Dispersitătsgradkoeffizient also ausdrücken:

$$
\mathrm{U}=\frac{\mathrm{P}}{\mathrm{L}} \cdot \mathbf{Z}
$$

Die Substanz, die als Niederschlag bei durchaus wahrnehmbarem $\mathrm{L}$ ausfallt, kann sich doch in sehr hochdisperser Form ausscheiden, wenn die Substanz in der Losung ein sehr großes Molekulargewicht besitzt (die Kompliziertheit der Zusammensetzung, Assoziation, Polymerisation, Solvatation usw.), weil die großen Moleküle sich langsam in der Flüssigkeit bewegen und bei Konzentrationsvergrößerung sich zu kleineren Gruppen verbinden, welche praktisch unfähig sind, die innere Reibung des Lösungsmittels $z u$ überwinden und sich, $\mathrm{nach}$ Auspressung des letzteren, mit einander $z u$ grobdispersen Teilchen … zu Kristallen zu vereinigen. Die vektoriale Kohäsion der Moleküle solcher hochmolekularen Körper ist unbedeutend, weil der Hauptteil der Energie auf die komplizierte innere Struktur verwandt worden ist; bei einer unbedeutenden Kohäsion kann man aber auch die Bildung eines dichten und stabilen kristallinischen Raumgitters schwer annehmen, vielmehr die Entstehung eines weichen und $\mathrm{sch}$ wachen kristallinischen Gitters (einem Schwamm ahnlich) erwarten, welches nit dem Lõsungsmittel durchtränkt und stark deformiert ist. Die das Gitter zusammensetzenden Teilchen sind aber ebenso vektorial, wie die Teilchen, welche das Gitterder gewönlichen Kristalle ausmachen und die Krafte, diedie Teilchen beieinanderzusammen-

1) Koll.-Zeitschr. 3, 286 (1908); 4, 138 FuBnote 74 (1909).

2) Auch die Solvatation. 
halten, sind in beiden Fälen vektorialer Natur. Hierin liegen, meiner Meinung nach, die Gründe für die Entstehung von Gallerten und Glästrn von löslichen hochmolekularen Substanzen').

Wenn wir den Einflub der Assoziation oder des großen Molekulargewichts auf den Dispersitätsgrad des Niederschlages bestimmen wollten, so müBten wir die Werte dieses Faktors bestimmen, welche äquivalent den Werten des Faktors $\frac{P}{L}$ sind; bezeichnen wir diese Werte mit $K$, so erhalten wir für den Dispersitătsgradkoeffizient folgende Formel :

$$
U=\stackrel{P}{L} \cdot Z \cdot K
$$

In Wirklichkeit ist der Ausdruck für $U$ noch komplizierter [vgl. Koll.Zeitschr. 3, 286 (1908)], aber ich will hier nicht năher darauf eingehen. Nicht selten überwiegt die Bedeutung von einem oder zwei Faktoren der Formel 3 dermaflen, daB man den EinfluB der anderen vernachläsšigen kann, und dann kann der Ausdruck für $U$ also vereinfacht werden:

$$
\begin{aligned}
\text { 1. } U & =\frac{P}{L} \\
\text { 2. } U & =\frac{P}{L} \cdot Z \\
\text { 3. } & U=Z \cdot K \text { usw. }
\end{aligned}
$$

Die erste Formel findet eine Anwendung bei Kondensationen in Mitteln, deren Viskosität sich wenig voneinander unterscheidet, und unter der Bedingung, da $B$ die Viskosität sich wenig mit der Konzentration verändert; die zweite Formel betrifft alle Fălle der Herstellung von dispersen Niederschlägen der Zusammensetzung nach einfacher und mit zunehmender Konzentration wenig assozilerender Substanzen; die dritte Formel bezieht sich auf die Erscheinungen des Gelatinierens hochmolckularer Körper. Genau genommen muB man bei einem vollständigen Studium des Konzentrationseinflusses auf den Dispersitatsgrad von Niederschlägenverschiedener Substanzen dieVeränderungen aller drei Faktoren - $\frac{\mathrm{p}}{\mathrm{L}}, \mathrm{Z}$ und $\mathrm{K}$ - im Auge haben.

Zum SchluB dieses Abschnitts wili ich besonders bemerken, da $B$ der Dispersitatsgradkoeffizient von mirzudem Zweck eingeführt worden ist, um die Kondensationsresultate in verschiedenen Dispersionsmitteln und verschiedener

1) Koll.-Zeitschr. 7. 241 (1910) und 9, 25 (1911). Wo. Pa uli, Koll.-Zeitschrift 7, 241 (1910). 
Substanzen bei Kondensationsgeschwindigkeiten vergleichen zu können, die sich unseren Wahrnehmungen entzieben. Da der fast "momentanen" Entstehung der dispersen Phase stets längere oder kürzere Zeit andauernde Veränderungen ihrer Dispersität folgen, so müssen wir uns jetzt mit den Gründen der Veränderung des Dispersitätsgrades von Niederschlägen mit der Zeit bekannt machen.

\section{Verănderungen der dispersen Niederschlăge mit der Zeit.}

Aus durchaus verständlichen Gründen ist es bis jetzt nicht gelungen, die Entstehung von Kristallkeimen zu beobachten, das Wachstum der Kristalle dagegen ist quantitativ genügend erforscht worden. Erst dcnn wird es möglich, die Entstehung der Kristallkeime zu verfolgen, sobald wir in der Lage sein werden, die Moleküle wahrzunehmen, denn das in bestimmte physikalisch-chemische Bedingungen gebrachte Molekül selbst stellt schon einen tatsăchlichen Kristallkeim dar 1). Bei konstanter Temperatur und Druck werden bei einer gewissen Konzentration ${ }^{2}$ ) solche Bedingungen ${ }^{8}$ ) an einer oder mehreren Stellen der Losung geschaffen, bei denen die den. Molekülen der gelösten Substanz eigenen vektorialen Kräfte den desorientierenden Einfluß der Moleküle des Lösungsmittels überwinden und die zusammenstoßenden Moleküle der gelösten Substanz zu mehr oder weniger regelrecht orientierten dispersen Teilchen vereinigen, welche dann infolge Wachsens deutliche Kristalle ergeben ${ }^{4}$ ). Bevor noch solche zum weiteren Wachsen fahige disperse Teilchen auftreten, können natürlich auch kleinere Teilchen entstehen, welche unter dem Einflub von desorientierenden Kräften des Lösungsmittels in cinzelne Moleküle zerfallen. Ein derartiger Kampf zwischen den orientierenden Kräften der gelösten Moleküle und den desorientierenden Kräften der Moleküle des Lösungsmittels kann sehr lange dauern; schlieblich müssen aber die gejösten Moleküle den Sieg davontragen, und dann entsteht das zum weiteren Wachsen fähige disperse Teilchen (für unsere Sinnesorgane erfolgt oft diese Entstehung, momentan"). In einfacheren

1) Das erste Stadium des Keimzustandes des Kristalis.

8) Diese Konzentration wird, abgesehen von der Loslichkeit der Substanz, noch durch eine ganze Reihe von in System herrschenden Granden bestimmt, von welchen die , In hom ogenität aller Art en * eine besondere Rolle spiclen.

8) Die ,Inhomogenitat" des Systems veranlaßt diese Bedingungen.

4) Vgl. meine Abh. "Zur Systematik des Aggregatzustandes der Materie ${ }^{*}$ in d. Kolloidchem. Beih. 
Fällen') wird die Wachstumsgeschwindigkeit eines derartigen Teilchens durch die Formel

$$
\mathrm{V}=\frac{\mathrm{D}}{\delta} \mathrm{O} \cdot\left(\mathrm{Q}^{1}-1\right)
$$

ausgedrückt, worin $\mathrm{D}$ den Diffusionskoeffizient, $\delta$ die Dickse der adhärierenden Schicht, $O$ die Oberfläche des Teilchens, 1 die Löslichkeit des Teilchens, $Q^{1}$ die Konzentration der Lösung, in der das Wachsen des Teilchens erfolgt, bezeichnet. Wahrend des Wachstumsprozesses des Teilchens wird 1 allmăhlich kleiner bis zur praktisch konstanten Gröhe L, was praktisch dann erfolgt. wenn das Teilchen schon die mittleren mikroskopischen Dimensionen erreicht. Sobald die Konzentration der Lösung $Q^{1}$ gleich $L$ wird, so hört das Wachsen des Teilchens auf. Auf diese Weise verringert sich die Wachstumsgeschwindigkeit im Laufe der Zeit bis auf 0 ; deshalb wird weiterhin von einer mittleren Wachstumsgeschwindigkeit bei der einen oder anderen anfănglichen Konzentration der Lösung die Rede sein. Aus dessen Formel (4) geht hervor, dat das Wachsen der dispersen Teilchen einer beliebigen Substanz äuBerst langsam verlaufen kann bei genügend kJeinem $Q^{1}-1$. Auf Grund dessen konnte es scheinen, als ob für einen jeden Körper, sogar für einen leicht Jöslichen, eine lăngere Dauer von hochdispersen Systemen möglich wäre, sobald $Q^{1}-1$ genügend klein ist; aber in Wirklíchkeit verhält es sich infolge der Uebersättigung und einer im Vergleich $z u$ den grobdispersen Teilchen gröBeren Losslichkeit der hochdispersen Teilchen anders.

Zeitweilig wollen wir die Veränderlichkeit der Löslichkeit mit der Dispersität außer acht lassen und annehmen, daB dieselbe konstant und gleich $L$ ist, dann haben wir für die Analyse des Kondensationsprozesses folgende Formeln:

$$
\left.\mathrm{U}=\frac{\mathrm{Q}-\mathrm{L}}{\mathrm{L}} ; \mathrm{V}=\frac{\mathrm{D}}{\delta} \cdot \mathrm{O}\left(\mathrm{Q}^{1}-\mathrm{L}\right)^{2}\right)
$$

Zur Klärung der Frage wollen wir die Kristallisationserscheinungen von $\mathrm{NaCl}$ und $\mathrm{BaSO}_{4}$ aus wässerigen Lösungen verfolgen. Der Einfachheit wegen wollen wir die LöBlichkeit von $\mathrm{NaCl}$ in Wasser auf $40 \mathrm{~g}$ in $100 \mathrm{ccm}$ und die von $\mathrm{BaSO}_{4}$ auf $2.10^{-4} \mathrm{~g}$ in $100 \mathrm{ccm}$ abrunden. Wenn wir nun auf die eine oder andere Art übersättigte Lösungen beider Substanzen herstellen, so sehen wir, daß es möglich

1) Ueber komplizierte Fälle vgl. die Arbeiten von R. Marc, Zeitschr. f. phys. Chem. 1910 ff.

2) $Q$ und $Q^{1}$ sind ungleich, namilich $Q^{1}<Q$, da dle Konzentration $Q$ infolge der Bildung der Kristallisationszentren bis $Q^{1}$ herabgeht. 
ist ${ }^{1}$ ), von $\mathrm{NaCl}$ Lösungen mit einer absoluten Uebersättigung ( $Q$ - L) bis zu $8 \mathrm{~g}$ ohne eine "momentane" Entstehung von Kristallisationszentren $z$ u bereiten, wahrend bei $\mathrm{BaSO}_{4}$. [z. B. bei der Reaktion $\left.\mathrm{Ba}(\mathrm{CNS})_{2}+\mathrm{MnSO}_{4}=\mathrm{BaSO}_{4}+\mathrm{Mn}(\mathrm{CNS})_{2}\right]$ die absolute Uebersättigung von $0,03 \mathrm{~g}$ nur Bruchteile einer Sekunde dauert. Beim $\mathrm{BaSO}_{4}$ tritt bei einer derartig minimalen absoluten Uebersāttigung wie $0,002 \mathrm{~g}$ eine deutliche Opaleszenz nach ca. 3 Stunden auf, eine $\mathrm{NaCl}$-Lösung ist bei gleicher absoluter Uebersättigung fast grenzenlos stabil.

Da für die Geschwindigkeit des Diffusionswachstums von schon entstandenen Zentren die absolute Uehersättigung von Wichtigkeit ist, so werden bei spontaner Aufhebung der Uebersättigung der $\mathrm{NaCl}$-Lösungen die Kristallisationszentren durch Diffusion bedeutend schneller wachsen als bei $\mathrm{BaSO}_{4}$, sogar in dem Fall, wo die relative Uebersättigung $\left(\begin{array}{c}Q-L \\ \mathrm{~L}\end{array}\right)$ für diesen Körper $\left(\frac{0,002}{2.10^{-4}}=10\right)$ ist"! Auf Grund dieses kann man behaupten:

1. Färgut lóslicho Substanzen kann man, wenn nur die Diffusion aichterschwert ist, keine stabilen hochdispersen Systeme im Gebiet der geringen relativen Uebersätigungen erhalten, weil die spontane Entstehung von Kristallisationszentren bei verhaltnismäBig hohen Werten der absoluten Uebersätigungen beginnt; das letztere bedingt aber das schnelle Diffusionswachstum der im ersten Moment hochdispersen Kristallisationszentren.

2. Für wenig lösliche Substanzen kann man hochdisperse-Systeme im Gebiet der verhältnismäbig geringen relativen Uebersättigungen erhalten, weil die spontane Entstehung von Kristallisationszentren bei sehr niedrigen absoluten Uebersattigungen beginnt; das letztere bedingt aber ein sehr langsames Diffusionswachstum der hochdispersen Kristallisationszentren.

Es ist leicht zu verstehen, daß wenn für baSO bei geringen relativen Uebersăttigungen (his 4) das Wachstum der Zentren einige zehn Stunden dauert. für Körper von verschwindend geringer Löslichkeit das Wachstum der Zentren bei relativen sogar 100 gleichen Uebersättigungen sich Jahrhunderte hirziehen kann. Wichtig ist es

1) G. Jaffé, Zeitschr. f. phys. Chem. 23, 565--594 (1903).

4) Vgl. .Grundzäge d. Dispersoidehem. 50-51. 
zu bemerken, dab im allgemeinen') bei gleichen relativen Uebersättigungen die Lösung eines leicht löslichen Körpers weniger stabil ist, als die eines wenig löslichen, was durch die unverhăltnismäBig große absolute Uebersattigung im ersten Fall hervorgerufen wird; der letztere Umstand aber setzt, wie es vom molekularkinetischen Standpunkt aus leicht zu begreifen ist, sehr stark die Stabilität des Systems herab, weil die Entstehung von Kristallisationszentren nach Maßgabe der Zunahme der absoluten Anzahl von Molekülen an Wahrscheinlichkeit gewinnen muB, welche sich in der Lösung im Vergleich zu der gewöhnlichen Sättigungskonzentration in grőßerer Menge befinden. Aus dieser Deduktion, der ein reirhes Versuchsmaterial entspricht, ist für uns von W'ichtigkeit die Schlubfolgerung, daB bei gleichen relativen Uebersăttigungen die Anzahl der Kristallisationszentren bei einem leicht löslichen Körper gröber ist, als bei einem wenig löslichen. Leider ist es äuberst schwierig (wenn nicht ganz unmoglich) eine genaue quantitative wechselseitige Beziehung zwischen der Anzahl der Zentren in heiden Fällen festzustellen, weil wir bei Körpern in der Art von $\mathrm{BaSO}_{4}$ einige Minuten hindurch eine $8-10 \mathrm{mal}$ übersättigte Lösung haben können, was bei Substanzen, deren .Löslichkeit einige zehn Gramm betrăgt, vollståndig undenkbar ist. Aus der Tatsache, dab man bei gleichen relativen Uebersăttigungen von Körpern geringer (jedoch verschiedener) Löslichkeit Niederschläge erhält, welche hinsichtlich der Dispersität sich nicht besonders voneinander unterscheiden, kann man annehmen, daG die Zahl der Zentren bei gleichen relativen Uebersättigungen bis zu einem gewissen Grade proportional der Löslichkeit der Substanz ist.

Aus allem Angeführten geht hervor, dab für wenig lösliche Substanzen bei spontaner Kristallisation ein unmittelbar an das Gebiet der stabil-übersăttigten Lósungen grenzendes Gebiet existiert mil verhältnismäBig kleinen Werten $U=\frac{Q-L}{L}$, in dem die hochdispersen Systeme verhältnismäBig lange dauern dank der Wachstumslangsamkeit der dispersen Teilchen oder dank den geringen Werten $Q^{\prime}-L$; bei leichtläslichen Substanzen ist aber die Existenz von hochdispersen Teilchen in diesem Gebiet von äußerst kurzer Dauer. Es gibt für wenig lösliche Körper noch ein zweites Gebiet, nämlich, das der sehr großen

1) Als Ausnahmen bis zu eincm gewissen Grade erscheinen die Falle der Ueberstittigung, welche durch die Existenz von mehreren Hydratformen, olymorphie u. a. hervorgerufen werden. 
Werte $U=\frac{Q-L}{l}$, in dem der hochdisperse Zustand bedeutend stabiler ist, als in dem eben erwähnten; diese Stabilität im zweiten Gebiet wird aber durch ganz andere Gründe bedingt. Bei der Verwirklichung der "momentanen " Entstehungsreaktionen von schwer löslichen Körpern, wie z. B. von $\mathrm{BaSO}_{4}$ durch die Reaktion

$$
\mathrm{Ba}(\mathrm{CNS})_{2}+\mathrm{MnSO}_{4}=\mathrm{BaSO}_{4}+\mathrm{Mn}(\mathrm{CNS})_{2}
$$

können wir die Entstehung im Innern der Lösung von eituigen zehn Gramm einer Substanz veranlassen, welche sich nur eine unermeBlich kurze Zeit hindurch in molekularer Zerteilung befindet und sich darauf "momentan" zu zahllosen feinsten dispersen Teilchen vereinigt, hierbei bleibt in der Lósung so weriig von molekular-gelöster Substanz, daß dieselbe durchaus nicht genügt für eine irgendwie wahrnehmbare Verringerung des Dispersitätsgrades des im ersten Moment entstandenen Niederschlages. Da die Löslichkeit eines hochdispersen Niederschlages gröter ist als die eines grobdispersen, so verändern sich im Laufe der Zeit die Niederschläge, welche im Gebiet der L.ösungen bei durch 10.000 meBbaren Werten von $U=\frac{Q-L}{L}$ erhalten werden, ebenso wie im Gebiet von kleinem $U$. Zwei Gründe verlangsamen den Prozeb der Dispersitătsverringerung: Der erste Grund ist dem Wesen nach der nämliche, wie auch im Gebiet der kleinen U-Werte. Bezeichnen wir bei einer leicht- und schwerlöslichen Substanz mit $l_{1}$ und $l_{2}$ die Lisslichkeiten in hochdispersem Zustande, mit $L_{1}$ und $L_{2}$ die Löslichkeiten in grobdispersem Zustande, so mub der Bedeutung des Einflusses der Zerkleinerung auf die Dispersität entsprechend zwischen den angeführten Gröben eine wechselseitige Beziehung existieren, welche sich bis $z u$ cinem gewissen Grade

$$
\frac{l_{1}}{L_{1}}=\frac{L_{2}}{L_{2}}
$$

$\mathrm{n}$ ïhert. Aus diesem Verhültnis folgt, daB, wenn die Löslichkeit $L_{2 .}=10^{-4}$, so $l_{2}=2.10^{-4}$ (ein ähnliches Verhältnis ist von $G$. Hulet t experimentell für $\mathrm{BaSO}_{4}$ festgestelli), und wenn $\mathrm{L}_{1}=1$, so $\mathrm{l}_{1}=2 \mathrm{~g}$.

Die Umkristallisationsgeschwindigkeit wird, wenn man den Diffusionskoeffizient, die Oberflachen u. a. als annthernd annimmt. durch dir Ausdrücke bestimmt:

$$
\begin{aligned}
& \mathrm{I}_{1}-\mathrm{L}_{1}=1 \\
& \mathrm{l}_{2}-\mathrm{L}_{2}=10^{-4}
\end{aligned}
$$

hieraus ersieht man, dafs es für eincu leichtlöslichen Körper nicht möglich ist, wenn die Diffusion nicht exschwert ist, hochdisperse Systeme von irgend welcher l)auer auch bei 
möglichst großen U-Werten zu erhalten. Es muB gleich bemerkt werden, daB für leichtlösliche Körper sogar $U=1000$ faktisch undenkbar ist, denn, wenn man die Löslichkeit nicht vertingert, müßten für einen Körper $L=10 \mathrm{~g}$ in $100 \mathrm{ccm}$ Lösung $10000 \mathrm{~g}$ Substanz sich bilden und ausscheiden; für minimal lösliche Körper kann $U==$ Millionen sein.

Der zweite und sehr wichtige Grund liegt in der sehr starken Verunreinigung der hochdispersen Niederschläge bei ihrer äußerst schnellen Entstehung bei großen U-Werten ${ }^{1}$ ). Bei leichtlöslichen Körpern spielt dieser Grund eine verhältnismätig geringe Rolle, wenn er nur nicht die Diffussionsgeschwindigkeit beeinflubt, weil die Beimengungen (die Rede ist von leichtlöslichen) leicht ausgewaschen werden; bei wenig löslichen Körpern ist aber der Grund von Bedeutung. Indem die fremden Moleküle in die wenig löslichen dispersen Teilchen eindringen, stören sie erstens die Regelmäßigkeit der Orientierung der Moleküle, zweitens verringern sie die naktive" OberflächengröBc, d. h. bei gleicher Teilchengröße besteht die Oberfläche des verunreinigten Teilchens nicht mehr ausschlieblich aus Molekutlen der reinen Substanz. Beide Einflüsse der Verunreinigung verlangsamen die Umkristallisation, weil es klar ist, dak die bis zur verunreinigten Oberfläche vorgedrungenen Moleküle länger einen passenden Ort suchen müssen, um sich anzuklammern, als an einer vollkommen reinen Oberfläche. Durch die Verunreinigung wird die Umkristallisation so verzögert, daB z. B. die im Gebiet der großen $\mathrm{U}$-Werte erhaltenen $\mathrm{BaSO}_{4}$-Niederschlăge die hohe Dispersität länger als ein Jahr beibehalten, die reinen hochdispersen Niederschläge kristallisieren sich dagegen um. Wenn man sogar gallertartiges $\mathrm{NaCl}$ (aus Amylalkohol) der Einwirkung von Feuchtigkeit aussetzt, so kristallisiert dasselbe sehr langsam un und ergibt (wenn man wenig Wasser nimmt) die allerunregelmäBigsten Kristallbildungen. Durch Aenderung des Wasserquantuuss erhält man cine ganze Reihe von Uebergangsstufen vom „an.orphen "Zustande durch mibgestaltete Kristallbildungen bis zu regulären holoedrischen Kristallen. Es gibt also für wenig lösliche Körper zweiGebiete einer anhaltenden Existenz horhdisperser Systeme: Das erste Gebiet bei kleinen U-Werten, das zweite bei groben: im zweiten Gebiet dauern dic dispersen Systeme länger, weil autier dem geringen Wert der absoluten Uebersätigung bei der

3) Vgl. , Grundziige ${ }^{4} 97-98$. 
Umkristallisation auch noch die Verunreinigung der dispersen Teilchen einen hemmenden Einflub hat. ${ }^{2}$ ) Zwischen diesen beiden Gebieten befindet sich ein Gebiet von weniger dispersen Systemen, weil die absolute Uebersättigung bei ihrem Wachstum größer und der Einfluß der Verunreingung bei der Umkristallisation nicht so grob ist. Dic drei bezeichneten Gebiete haben wir bei der Kristallisation eines beliebigen Körpers von beliebiger Löslichkeit; aber abhängig von der letzteren sind diese Gebiete von verschiedener Ausdehnung hinsichtlich der absoluten Konzentration und hinsichtlich der Existenzdauer des hochdispersen Zustandes. Bei leichtlöslichen Körpern ist von längster Dauer hinsichtlich der absoluten Konzentration das mittlere Ciebiet, weil man bei einer Ausscheidung von einigen zehn Gramn große Kristalle erhält; das erste und dritte Gebiet sind von kurzer Dauer. Bei Körpern von minimaler Löslichkeit fließen alle drei Gebiete zusammen, denn in den beiden ersten Gebieten ist die absolute Uebersättigung, auf deren Kosten die dispersen Kristallisationszeniren wachsen könnten, so gering, daß man das Wachsen erst im Laufe von vielen Jahren wahrnimmt; im letzten Gebiet geht die Veränderung der Dispersität noch langsamer, weil der hemmende Einflub der Verunreinigung hinzukommt. In bezug auf die absolute Konzentration sind bei $\operatorname{seh} r$ wenig löslichen Körpern die beiden ersten Gebicte sehr eng und umfassen zusammen eine Konzentration von einigen Bruchteilen eines Milligramms, dafü: kann aber das dritte Gebiet eine Konzentration von einigen zehn Gramm haben.

Als ein bemerkenswertes Beispie! der letzteren Kategonit vin Körpern erscheint $\mathrm{CuCl}_{2}$ in Benzol, in dem die Löslichkeit desselben fast 0 ist. Leitet man zur Darstellung von $\mathrm{CuCl}_{\mathrm{z}}$ in $\mathrm{Renzo]}$ die Reaktion zwischen oleinsaurem Kupfer und $\mathrm{HCl}$ eirı, so ertäit man bei allen analytischen Konzentrationen, sowohl bet ganz yeringen als such äußerst grolen gallertartige $\mathrm{CuCl}_{2}-\mathrm{Fl}$,cken, welche aus in günstigsten Fall an der inikroskopischen Selugrenze liegenden Teilchen bestehen. (P. P. von Weinarn li. J. B. Kanan.) Der Unterschied $z$ wischen den beiden angefürten Fällen eines leichtlöslichen und thes minim löslichen körpess liegt nur in der quantitativen Richtung der Kristallisationserscheinung und durchaus nicht in der quali.

7) Außerdam muR man in Gedachtnis halten, daB das Verhültuis der Lah! der dispersen Teikcheri zam gelosten Teile bei großen U-Werten unvergleichbs: grbaur als bei kitinen $\mathrm{U}$-Werten ist. 
tativen: wenn wir in der Lage wäreneinenganzen See einer $\mathrm{CuCl}_{z}$ - Lösung von verschwindend geringer Konzentration herzustellen und ihn Jahrhundertehindurch $z u$ beobachten, so würden wir auf dem Grundegrobe $\mathrm{CuCl} \mathrm{Cl}_{2}-\mathrm{Kr}$ istalle finden. Zur Erlauterung will ich kurz die Dispersitätsgradänderungen eines $\mathrm{BaSO}_{4}$-Niederschlages bei fortschreitender Zunahme des Wertes

$$
\mathrm{U}=\frac{\mathrm{Q}-\mathrm{L}}{\mathrm{L}} \cdot \mathrm{K} \cdot \mathrm{Z}
$$

in der Reaktion $\mathrm{Ba}(\mathrm{CNS})_{2}+\mathrm{MnSO}_{4}=\mathrm{BaSO}_{4}+\mathrm{Mn}(\mathrm{CNS})_{2}$ untersuchen. Vorher möchte ich aber auf die Gründe hinweisen, welche mich bewogen haben in den Dispersitätsgradkoeffizienten nicht die variable Grobe der Löslichkeit (1) einzuführen. Erstens ist die Gröbe 1 unbekannt und ihre Bestimmung experimentell entweder mit großen Schwierigkeiten verknüpft (vgl. die Arbeit von G. Hulett) oder füi: sehr hochdisperse Teilchen überhaupt unmöglich; die Gröbe $L$ dagegen ist in der Mehrzahl der Fälle bekannt oder kann in vielen Păllen durch die elektrische Leitfühigkeit der Lösung bestimmt werden. Zweitens können, da die Zunahme der Losslichkeit mit dem Dispersitătsgrad annăhernd proportional zu der Losslichkeit der Substanz in grobdisperser Form erfolgen muB, d. h. da annähernd die wechselseitige Beziehung $\mathrm{l}=\mathrm{kL}$ beobachtet wird, worin $\mathrm{k}$ gröBer als $\mathrm{I}$ ist, keine besonderen Vorteile durch die Einführung von 1 statt $\mathrm{L}$ in die Formel entstehen. Drittens endlich erreicht das disperse System einen Gleichgewichtszustand bei $Q-L=0$ und nicbt bei $Q-1=0$. Man hat überhaupt keinen Grund $L$ durch $I$ zu ersetzen, weil für die Analyse von anhaltenden Aenderungen des Dispersitatsgrades durch das Diffusionswachstum der "momentan * entstandenen dispersen Kristallisationszentren wir die Formel besitzen:

$$
\mathrm{V}=\frac{\mathrm{D}}{\delta} \cdot \mathbf{0}\left(\mathrm{Q}^{\prime}-1\right)
$$

Kehren wir zu der Untersuchung des Einflusses der Zunahme von $U$ auf den Dispersitätsgrad von $\mathrm{BaSO}_{4}$ zurück, so muB bemerkt werden, daB mit zunehmender Konzentration der reaglerenden $\mathrm{MnSO}_{4}$ und $\mathrm{Ba}(\mathrm{CNS})_{2}$-Losungen sowohl die Viskosität derselben als auch der Assoziationsgrad (das Tyndall phänomen bei groBen Konzentrationen) zunimmt; diesen Einflul aber durch Zahlen auszudrücken, ist augenblicklich noch schwierig, deshalb habe ich mich auf die Anwendung des vereinfaciten Ausdrucks:

$$
\mathrm{U}=\frac{\mathrm{Q}-\mathrm{L}}{\mathrm{L}}
$$


beschränkt; nimmt man der Einfachheit wegen $L=2.10^{-4}$ an, so erhalten wir für die drei erwähnten Gebiete folgende Ausdelınungen in den relativen und absoluten Konzentrationen:

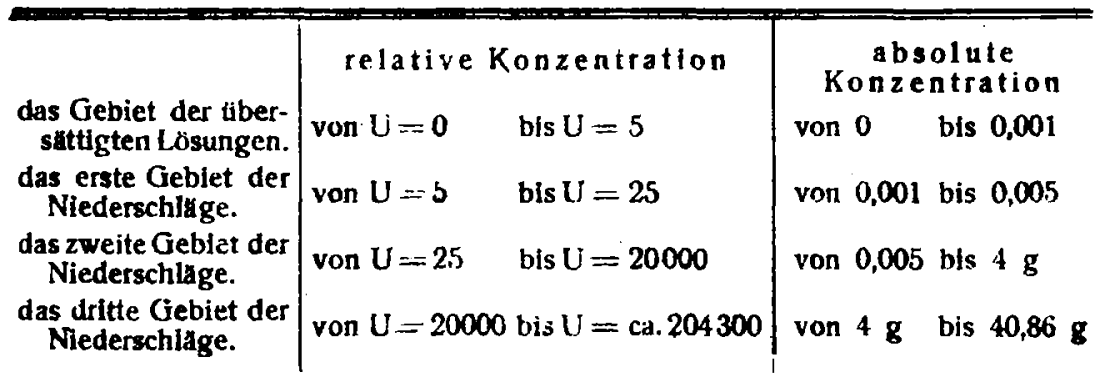

Aus dieser Tabelle sieht man, daB das erste Gebiet's der feindispersen Niederschlăge (welche die ziemlich große Dispersität dank dem langsamen Wachsen der dispersen Teilchen von einigen Stunden bis zu cinigen Minuten beibehalten! eine absolute Konzentration von nur $0,004 \mathrm{~g}$ umfaBt; das Gebiet der fast "momentan * entstehenden grobdispersen Niederschlăge umfaBt ca. $3 \mathrm{~g}$; dagegen das dritte Gehiet der "momentan" entstehenden hochdispersen Niederschläge umfaBt um $37 \mathrm{~g}$ herum. Aus diesen Daten ersieht man sofort, dab, wenn wir künstlich die Löslichkeit von $\mathrm{BaSO}_{4} 10^{5} \mathrm{mal}$ verringern, die beiden ersten Gebiete der dispersen Systeme zu solchen minimalen absoluten Konzentrationen sich verschieben, welche jenseits unserer gewöhnlichen analytischen Konzentrationsgrenze liegen; die absolute Konzentration der beiden ersten Gebiete wiral sich nămlich zwischen $1.10^{-8}$ und $4.10^{-5} \mathrm{~g}$ befinden. Wenn bei $\mathrm{BaSO}_{4}$ in Wasser die hochdispersen Teilchen durch Diffusion z. B. im Laufe einer Stunde bis zu deutlichen holoedrischen Kristallen heranwachsen; so werden bei einer $10^{5} \mathrm{mal}$ verringerten Löslichkeit von $\mathrm{BaSO}_{4}$ zu demselben $\mathrm{Z}_{\text {weck }}$ mehr als 10 Jahre erforderlich sein. Das dritte Gebiet aber wird sich bei einer Löslichkeitsverringerung in der absoluten Konzentration etwas ausdehnen und in der relativen wächst es ungeheuer an, weil $U$ bis $2.10^{10}$ vergröBert werden kann. Der Versuch zeigt in der Tat, daB bei der $\mathrm{BaSO}_{4}$-Entstehung in starkem wässerigem Alkohol man bei allen erreichbaren Konzentrationen hochdisperse Niederschlăge erhält, welche sogar nach 3 Jahren nicht nur keine wahrnehmbaren Kristalle ergeben, sondern auch sehr wenig in dieser Zeit ihre Dis-

1) Das erste Gebiel beginnt wahrscheinlich schon früher, aber im Hinblick auf die geringe Konzentration der sich ausschpidenden dispersen Phase gelingt es nicht, den Beginn wahrzunehmen. 
persität verändern. Man hat nicht den geringsten Grund daran zu zweifein, daß, wenn wir nur freier über Zeit und Volumen der Lösungen verfügen könnten, auch bei fast vollkommen unlöslichen Körpern der Grundsatz Geltung haben würde: mit zunehmendem U-Wert vergröBert sich auch der Dispersitätsgrad der erhaltenen Systeme - oder besser gesagt: mit steigendem U-Wert entstehen Systeme mit zunehmendem Vorrat an freier Oberflächenenergie ${ }^{1}$ ). Die letztere liormulierung hat für die kristallinischen dispersen Niederschläge aus folgendem Grunde ihre Vorzüge: bei schneller Kristallentstehung wachsøn die letzteren in den Richtungen der größten Wachstumsgeschwindigkeiten und im Resultat erhalten wir Kristallskelette und Nadeln; diese Bildungen sind bei mit den holoedrischen Kristallen (welche bei langsamer Kristallentstehung erhalten werden) gleicher spezifischer Oberfläche doch weniger stabil; weil sie über einen größeren Vorrat an freier Oherflächenenergie verfügen. Wollen wir den eben ausgesprochenen Satz an einem Beispiel - $\mathrm{BaSO}_{4}$ in Wasser - erläutern. Verändern wir progressiv $U$ und begnügen uns mit kurzfristigen (z. B. $1 ; 4-1 / 2$ Stunde) Beobachtungen der dispersen $\mathrm{BaSO}_{4}$-Systeme, so nehmen wir mit voller Deutlichkeit das Vorhandensein eines Minimums der Dispersität wahr, weil die Kristallisationszentren im ersten Gebiet der Niederschläge langsam wachsen ${ }^{2}$ ). Bei anhaitenden Beobachtungen wird dieses Minimum weniger deutlich. Wenn wir schlieBlich den Versuch so führen, daß wir bei geringen Konzentrationen bedeutend gröBere Mengen der reagierenden Lösungen (man läßt den Staub sich absetzen) als bei großen Konzentrationen nehmen (um überzeugt zu sein, daB die kleinen Kristalle nicht aus. Mangel an Substanz in der Lösung bei geringen Konzentrationen und nicht durch den EinfluB des Staubes auf die Kristallisationszentren entstanden sind) und das

1) Ich muB noch einmal ausdrucklich hervorheben, daB dieser Satz fur das Endstadium des Kristallisationsprozesses gilt, d. h. wenn die Beobachtungen über den Dispersitătsgrad der Niederschläge nach der oft auBerordentlich langen Zeit gemacht werden, welche der vollständigen Beendigung des Kristallisationsprozesses entspricht. Bei kleineren Zeitintervallen wird das Minimum in den Dispersitatsgraden der Niederschlage mit geringerer Deutlichkeit hervortreten.

3) Widtig ist es $z u$ bemerken, daß auf die Zahl der Zentren im ersten Gebiet der Niederschlage der Staub einen großen Einfluß ausübt, er vergroßert numlich die Zahl derselben; im zwelten und dritten Gebiet spielt dieser Einflus fast keine Rolle. Die Zunahme der Kristallisationszentren erhoht die Dispersitat des Niederschlages. 
Ende des Wachstums der Kristalle bei geringen Konzentrationen abwarten, so wird es kein Minimum geben. Für $\mathrm{BaSO}_{4}$ ist mir das durch 6 Monate währende Beobachtungen gelungen festzustellen ivgl. meine Mikrogramme in d. Koll.-Zeitschr.). Aus dieser Spanne Zeit geht hervor, daß, um das Fehlen des Minimums beim $\mathrm{BaSO}_{4}$ mit $10^{5}$ mal verringerter Löslichkeit festzustellen, ein 7.eitraum erforderlich wäre, der das Menschenalter um einige Generationen bei weitem übertreffen würde.

Vielen mag es paradox erscheinen, daß man aus einer verdün rten $\mathrm{BaSO}_{4}$-Lösung einen größeren Kristall erhalten kann, als aus einer konzentrierteren. Das wird einem aber aus dem folgenden sofort klar: nehmen wir einige hundert Liter einer solchen übersättigten $\mathrm{BaSO}_{4}$ Lösung, in der im Laufe der Zeit nur ein Kristallisationszentrum entsteht, oder führen wir künstlich in dieselbe einen Kristallkeim ein, so wird dieses Zentrum sehr langsam wachsen, die ganze gelöste im Ueberflue befindliche Substanz augenscheinlich (wenn kein zweites Zentrum auftritt) sich an demselben ablagern und einen mit unbewaff netem Auge sichtbaren Kristall ergeben.

Es muB hinzugefügt werden, daß während des langsamen Wachsens der Kristallisationszentren im ersten Gebiet der Niederschläge auch đie Niederschläge in den beiden anderen Gebieten sich verändern, sie verringern nämlich ihren Dispersitätsgrad. Diese Verringerung des Dispersitätsgrades ist aber in diesem Gebiet bedeutend geringer, als im ersten aus Gründen, welche einem durch das $\mathrm{BaSO}_{4}$-Beispiel erklärlich werden. Die Vergrößerung des Volumens der reagierenden Lösungen hat nur im ersten Gebiet der Niederschläge einen Einfluß auf die Kristallgröße und einen um so grð̋eren bei solchen schwachen übersättigten Lösungen, in denen die Aufhebung der Uebersăttigung durch wenig zahlreiche Zentren sich sehr lange hinzieht bei einer verschwindend kleinen absoluten Substanzmenge in der Lösung. Im $z$ weiten Gebiet der Niederschläge, wo die Uebersättigung ,momentan ${ }^{*}$ aufgehoben wird, erhält man mikro-disperse Niederschläge (für $\mathrm{BaSO}_{4}$ ); die VolumenvergröBerung beeinflubt nicht in sichtbarer Weise die Kristallgröße. Im dritten Gebiet kann ebenfalls kein Einflub des Volumens auf den Dispersitätsgrad wahrgenomnen werden. Das zweite Gebiet der $\mathrm{BaSO}_{4}$-Niederschläge verändert verhältnismäßig in äußerst geringem Grade seine Dispersität, weil der Niederschlag aus mikroskopischen Körnern bestcht, deren Löslichkeit sich sehr wenig von der Löslichkeit der großen $\mathrm{BaSO}_{4}$-Kristalle unterscheidet. Das dritte Gebiet verăndert langsamer seine Dispersität, als die Niederschläge des 
ersten Gebiets, weil infolge der Verunreinigung der dispersen Teilchen die Umkristallisation gehemmt wird. Wenn wir die $\mathrm{BaSO}_{4}$-Niederschläge aller Gebiete bei einer konstanten Temperatur im Laufe einer längeren Zeit sich umkristallisieren lassen würden, so würden natürlich alle in einen grobdispersen kristallinischen Zustand übergehen; aber auch fïr diese groblristallinischen Niederschläge würde der oben angeführte Satz in folgender Fassung Geltung haben: Die gröberen Kristalle befinden sich in Lösungen, deren Konzentration gering war's.

Ich glaube, daß die angeführten Beispiele genïgen sowohl zur Erklärung der Verschiebung der drei Gebiete der dispersen Niederschläge hinsichtlich der "absoluten" und "relativen" Konzentrationen, als auch zum Verständnis der Dauer des Verringerungsprozesses des Dispersitätsgrades mit der Zeit. Für den Kolloidforscher ist es wichtig, dab die beiden ersten Gebiete der Niederschlage in die Gebiete von so geringen "absoluten "Konzentrationen sich verschieben, welche weit hinter den allerkleinsten erreichbaren Konzentrationen liegen. Dann werden wir in allen den Versuchen zugänglichen Gebieien hochdisperse Niederschläge haben, welche ihre Dispersităt nicht in möglichen Beobachtungsräumen bis zu deutlichen Mikrokristallen verringern, auber wenn man künstliche Mittel anwendet.

Hieraus folgt, dab ein im Sinne von Graham, typisches Kolloid"eine beliebige Substanz darstellt, welche aus einem dieselbe so minimallosenden Losungsmittel nieder. geschlagen ist, dab derForscher nicht die Moglichkeit hat, abzuwarten, bisdiehochdispersenkristallcheneine Gröbe erreicht haben, welche ihre Kristallform zu erkennen gestattet; die Substanz ist um so ,menr kolloid“, je ungünstiger die Lösungsbedingungen sind, weil dadurch schon die "absolute" Konzentration minimer wird, bei der man die Entstehung von deutlichen dispersen Kristallen erwarten kann. Als ,typisches Kolloid" in diesem Sinne erscheint $\mathrm{CuCl}_{2}$ in trockenem Benzol, im Wasser dagegen stellt es ein "t ypisches Kristalloid* dar.

\section{Die Entstehung von Kolloidlosungen und der Grund ihrer Stabilităt.}

Oben war die Rede von dem Einfluh des U-Wertes auf den Dispersitätsgrad des Systems, ohne uns dafür $z$ u interessieren,

1) Vgl. die vorhergehenden Anmerkungen. 
ob hierbei eine kolloide l.ösung entstehı oder nicht; wir haben nur festgestellt, daß man hei einem beliebigen in möglichst ungünstige Lösungsbedingungen gebrachten Körper hei allen heim Versuch möglichen U-Werten hochdisperse Systeme erhält'). Jetzı wollen wir der Frage, wann man kolloide Lösungen erhält, näher treten. Vorher möchte ich aber noch einmal ${ }^{2}$ ) die Aufmerksankeit darauf ienken, dab es ganz unmöglich ist, in einem, vollkommen indifferenten" Lösungmittel eine irgendwie stabilekolloidelösungbei einerirgendwiebedeutenden Konzentration zu erhalten, stets ist aber eine solche von äuberst geringer Konzentration möglich. Am verståndlichsten wird dieser Grundsatz vom molekularkinetischen Stand-

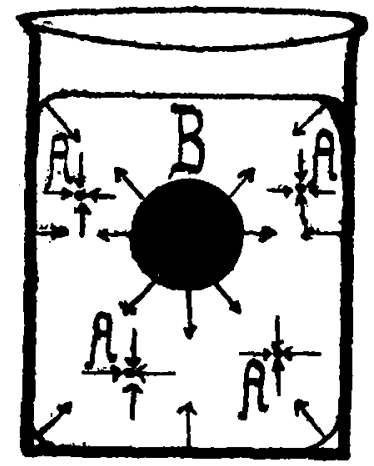

Fig. 10

A, A, A, A - die auBerst hochdispersen Teilchen.

B - das grobdisperse Teilchen.

punkt aus. Nehmen wir ein "vollkommen indifferentes." Lösungsmittel, und făhren wir in dasselbe äuberst hochdisperse Teilchen eines unlöslichen Körpers ein, so verrichten wir damit eine Arbeit gegen den normalen Druck des Lösungsmittels (s. Fig. 10 A, A, A, A . und diese wird um so größer sein, je größer die Zahl der Teilchen

1) Notwendig ist es natuirlich, daB bei allen U-Groben die Loslichkelt verschwindend klein bleibt: sehr hăufig kommt es vor, daB bei Reaktionen in groBen Konzentrationen die Loslichkeit stark zunimmt, dann kann man augenscheinlich $U$ nicht grob annehmen. Aehnliche Beispiele werden in den Mittellungen aus meinem laboratorlum veroffentlicht (P. P. von Weimarn und A. L.Stein); vgl. das SchluBwort dieser Abhandlung. Ebenso wichtig ist es, daA bei groBen Konzentrationen die Reaktionsgeschwindigkeit nicht gehemmt wird.

3) Vgl. den ersten Teil dieser Abhandlung in den Kolloidchem. Beih. 1, 412. 416 (1910) und Grundzüge d. Dispersoldechem. 90-93, 120-121. 
und je kleiner dieselben sind. Das letztere wird uns verständlich, wenn wir uns ins Gedächtnis' zurückrufen, daß auf die grobdis persen Teilchen der normale Druck überhaupt keine Wirkung ausübt, weil ihre Oberfläche gleichsam die Rolle von Wandungen im Innern eines Gefässes spielt, von welchen der normalc Druck seine Richtung (s. Fig. 10 B) ins Innere der Flüssigkeit einschlägt. Ein System, daß aus einer derartigen indifferenten Plüssigkeit mit eingeführten hoch dispersen indifferenten Teilchen besteht, wird über elnen gröberen Vorrat an freier Energie verfügen, als in einer reinen Flüssigkeit, und diese Energie wird um so gröber sein, je höher die Dispersität der eingeführten Teilchen, je gröber ihre Anzahl und je indifferenter die Flüssigkeit. Sobaid in einem solchen Systen keine die Verringerung der Dispersität der Teilchen hemmenden Faktoren obwalten, so wird dasselbe bestrebt sein, die Dispersität der eingeführten Teilchen aut irgend eine Art und Weise zu verringern. was in gegebenen Fall durch die "Koagulation" oder "Ausflockung" erfolgt. Aber wie stark das Streben nach einer Verringerung der Dispersität auch sein mag, so mub doch bei auberst geringen Konzentrationen eine durchaus wahrnehmbare $S p a n n e Z$ eit zum $Z_{\text {weck }}$ des $Z$.usammentreffens der von einander weit entfernten Teilchen verstreichen '). Unabhöngig von dieser theoretischen Erklärung ist der angeführte Grundsatz eine unmittelbare Folge des ganzen experimentellen Materials der derzeitigen Kolloidchemic.

Die bemerkenswerten Untersuchungen von A. Lottermoser ${ }^{2}$ ) zusammen mit den Arbeiten in derselben Richtung von anderen Gelehrten sprechen deutlich dafür, dab nur die in Bezıg auf die kolloiden Teilchen chemisch aktiven Ionen (welche in nicht so großer Konzentration hinzugefügt werden, daB dadurch die Löslichkeit stark vergrößert wird) diese Teilchen peptisieren, d. h. die Stabilität der Kolloidlösungen vergrößerṇ. Man kann mit. Bestimmtheit behaupten, daß gerade die $z ı$ den dispersen Teilchen indifferenten lonen besonders aktiv zum Lösungsmittel (Wasser) sind; sie schließen die Moleküle des Wassers an sich, machen das Lösungsmittel in bezug auf die dispersen Teilchen indifferenter und vergrößern außerdem den normalen

1) Wie bekannt, hat The Svedberg für die Methode der elektrischen Dispersion den Grundsatz aufgestellt, daB im vollständig passiven Mittel eine kolloide Lobsung "nicht existenzfähig * ist [Koll.-Zeitschr. 2, 149 (1907)]; ich glaube, daß der Satz so lauten müBle, wie oben von mir formuliert wurde.

2) Vgl. Koll.-Zeitschr. 4, $128 \mathrm{ff}$. (1909). Hier ist auch auf die Aikzandlungen von A. Lottermoser hingewiesen. 
Druck des Lösungsmittels. Das ist einer von den Gründen des koagulierenden Finflusses der Ionen.

In einer der nächstfolgenden Abhandlungen') (P. P. von Weimarn und J. B. Kahan) werden einige Beispiele für den hier aufgestellten Grundsatz des indifferenten Dispersionsmittels und der Stabilität der Kolloidlösungen angeführt; bemerken will ich noch, dab dieser (irundsatz auf so verschiedene Dispersionsinittel wie $z$. B. Benzol und Wasser anwendbar ist.

Damit die Kolloidlösung bei verhältnismäBig bedeutenden Konzentrationen stabil ist, ist ohne Frage erforderlich, daß der indifferente Zustand des Dispersionsmittels durch Mittel vermindert wird, welche nicht wahrnehmbar die lösende Fähigkeit des Dispersionsmittels erhöhen. Vielleicht habe ich im ersten Teil dieser Abhandlung ${ }^{2}$ ) diese Mittel zu verschleiert und allgemein formuliert ${ }^{3}$ ); solche Mittel sind sehr mannigfaltig, und ich will jetzt auf einige derselben hinweisen: 1. Das Einführen von molekulargelösten Stoffen in ein Dispersionsmittel bis zur Entstehung einer kolloiden Lösung; in diesem Fall verbinden sich die dispersen Teilchen sehr häufig infolge der Kapillar- oder Adsorptionskräfte mit den Molekülen der eingeführten Substanz und halten sich auf Kosten derselben (kolloider Parasitismus) länger im Dispersionsmittel, weil dasselbe zu den Molekülen der eingeführten Substanz nicht indifferent ist. 2. Das Einführen von solchen Substanzen, welche langsame chemische Umwandlungen der dispersen Teilchen in leichtlösliche Verbindungen hervorrufen, wobei die Menge der entstehenden Verbindungen nicht so bedeutend sein darf, daß die Löslichkeit der dispersen Teilchen merklich zunimmt. 3. Das Einführen von solchen Stoffen, um deren Besitz das Lösungsmittel und die dispersen Teilchen ringen würden; die Konzentration der eingeführten Substanzen mub gering sein, um nicht merklich die Löslichkeit der dispersen Teilchen $z u$ verändern, und damit nicht bald der Sieg der einen Scite rufällt. Als Beispiel für die Anwendung des ersten Mittels dient die "Schutzwirkung " der hochmolekularen Körper (C. Pa I und dessen Schüler); Beispiele für das zweite Mittel sind dic Peptisationsfälle unter dem Einfluf der

1) Siehe das Sclihbwort dieser Abhandlung.

2) Kolloidchem. Behl. 1, 396-422 (1910).

8) Dieser Ansicht ist z. B. Wo. Ostwald, (jtundriB; 2. Aufl. (Dresden 1911), 111-112, FuBnote 3. Aus AnlaB dieser Bemerkung muß ich darauf hinweisen, daB ich die mechanische, elektrische und $d$. ahnliche Methoden vom molekularkinctischen Standpunkt aus noch nicht im Druck besproch'n habe; meine Theorien betrafen bis jetzt nur dic physiko-chemischen Methoden. 
lonen (A. Lottermoser u. a). Für das dritte Mittel kann das System Benzol + die disperse Phase $\mathrm{CuCl}_{2}$ als Beispiel dienen (P.P. von Weimain und J. B. Kahanl); der Zusatz eines geringen Wasserquantums erhöht in sehr bedeutender Weise die Stabilităt der kolloiden Zösungen infolge des Kampfes zwischen dem Benzol und $\mathrm{CuCl}_{2}$ um das Wasser; setzt man Wasser in genügender Menge zur Bildung von (uCl. $\cdot 2 \mathrm{H}_{2} \mathrm{O}$ hinzu, so entstehen sehr instabile kolloide Lösungen dieses Körpers $\left.\left(\mathrm{CuCl}_{2} \cdot 2 \mathrm{H}_{2} \mathrm{O}\right)^{4}\right)$. Das Aufzählen aller moglichen Verschiedenartigkeiten der angeführten Mittel zur Erhöhung der Stabilität würde zu viel Zeit beanspruchen und ein ganzes Werk anfüllen, deshulb paBt es nicht in den Rahmen dieser Abhandlung.

Es muß in bezug auf alle Mittel zur Erhöhung der Stabilităt kolloider Lösungen noch folgendes bemerkt werden: Die Erniedrigung der Loslichkeit der kolloiden Phase infolge des Zusatzes einer die Stabilität der kolloiden Lösung erhöhenden Substanz darf nicht die stabilisierende Wirkung des Zusatzes kompensieren oder übertreffen; die VergröBerung der Löslichkeit der kolloiden Phase infolge des Zusatzes einer stabilisierenden Substanz darf keine irgendwie schnelle Umkristallisation und Auflösung der dispersen Teilchen hervorrufen. Diese Bedingungen sind experimentell leicht zu erfüllen, man braucht nur in dem einen oder anderen Sinne die Konzentration des Zusatzes zu verăndern.

SclılieBt man die "Schutzwirkung* der hochmolekularen Stoffe, welche gewöhnlich auch in großen Konzentrationen die Loslichkeit der dispersen Phase fast garnicht verändern, aus, so kann man folgenden idealen Grundsatz für die Erhöhung der Stabilităt kolloider l.ossungen aufstellen: die stabilisierende Substanz, welche fast gar nicht die minime Loslichkeit der kolloiden Phase verändert, mub das Dispersionsmittel nurdank dem Umstande weniger indifferent machen, dab in demselben sehr langsam aufhörende molekulare Störungen hervorgerufen werden. Das Beispiel des Systems $\mathrm{CuCl}_{3}+\mathrm{C}_{6} \mathrm{H}_{6}$, von dem früher die Rede war, nähert sich diesem idealen Grundsatz. Wenn keine stabilisierenden Faktoren vorhanden sind, so erhält man eine merkliche' Zeit hindurch dauernde kolloide L.̈̈sungen von minim losslichen Körpern bei gewöhnlich geringenen als

1) Bei groBen Wassermengen geht das System aus einem suspensoiden in ein emulsoides über und die Stabilität wird von neuem erhoht (P. P. von Weimarn und J. B. Kahan). 
$\frac{1}{100000}$ der normalen Konzentrationen; in diesen Fallen ist $U$ sehr grob und wir befinden uns im dritten Gebiet der Niederschläge, weil die beiden ersten Gebiete für wirklich verschwindend lösliche Körper hinter den Grenzen unserer Beobachtungsmittel liegen. Bei merklich löslichen Körpern (wie $\mathrm{BaSO}_{4}$ in Wasser) kann man kolloide Lösungen im ersten Gebiet der Niederschläge erhalten (vgl. meine früheren Abhandlungen); die Stabilität dieser Lösungen übersteigt, wenn in denselben eine merkliche Menge von dispersen Teilchen enthalten ist, selten einige Stunden, bei sehr geringer Konzentration der dispersen Teilchen sind die Lösungen dagegen stabil, es ist aber fast unmoglich sie zu beobachten, weil es äukerst schwierig ist, die Teilchen infolge ihrer undichten Verteilung im Dispersionsmittel wahrzunehmen.

Aus allem Gesagten ergibt sich die auf den ersten Blick paradox erscheinende Schlubfolgerung: Für ein „typisches') Kolloid, welches bei allen möglichen experimentell erreichbaren U-Werten nicht einmal in Form von feinsten deutlichen Mikrokristallen erhalten werden kann, ist es unmöglich, eine kolloide Lösung von bedeutender Konzentration herzustellen, wennim Dispersionsmittel nur keine stabilisierenden Prozesse stattfinden.

\section{Ausflockung.}

Wie bekannt, sammeln sich bei der Koagulation die dispersen Teilchen z.u Flocken von verschiedener Konsistenz; diese Flockenbildung nennt man "Ausflockung" (G. Malfitano l. c.). Diesen Prozeb kann man jurchaus nicht als charakteristisch für die Teilchen von kolloider Dispersität bezeichnen, weil wir schon aus dem auf den ersten Seiten dieser Abhandlung angeführten $\mathrm{NaCl}$-Beispiel deutliclı gesehen haben, daB die Flockenbildung auch den kleinen Mikrokristallen eigen ist. Ebenso wenig kann man die "Ausflockung" als eine Aenßerung von Kristallisationskräften ansprechen, weil die Ansammlung von Teilchen zu Flocken nicht durch dieselben hervorgerufen wird. Nur dann, wenn die Teilchen sich dem Wirkungskreise der Moleküle nähern, ist ein Einfluß von Kristallisationskräften möglich, und tatsăchlich äuBert sich auch ein solcher, welcher sich in einer Umformung der Teilchen zu gröBeren, zuweilen (in den Fällen, wo

1) In einem anderen Dispersionstnittel kann dieses Kolloid naturlich ein typisches Kristalloid* sein. 
die Löslichkeit nicht fast $==0$ ) zu holoedrischen Kristallen offenbart (P. P. von Weimarn, M. Traube-Mengarini und A. Scala. J. Amann). Es muB noch bemerkt werden, dab die Umformung der kolloiden Teilchen zu deutlichen Kristallen nur dann verhältnismåtig schnell erfolgt, wenn die Teilchen wenig verunreinigt sind und das Losungsmittel diese Umformung begünstigt (merkliche Löslichkeit).

\section{SchluBwort.}

Mit dieser Arbeit schliebt die Publikation aller theoretischen und experimentellen Daten, welche in den Rahmen der Fortsetzung der mit dem \$29 abgebrochenen Abhandlung "Zur Lehre von den $Z$ uständen der Materie " gehören'). Wie bekannit, wurde diese Abhandlung im Januar 1908') zu drucken begornen, und erst jetzt wird der Druck zu Ende geführt. Als Grund fär diese Verzögerung erscheint (abgesehen von einer Reihe von Zufälligkeiten rein persönlichen Charakters, welche alle meine ursprünglichen Pläne und Berechnungen umstießen) die Notwendigkeit in den Jahren 1908-1910 das Laboratorium der physikalischen Chemie am Berginstitut einzurichten und die Vorlesungen über physikalische Chemie. welche früher vollständig fehlten, einzufiuhren. Die letztere Arbeit ist von mir jetzt zu Ende geführt worden, und ich hoffe, daB die ferneren Veröffentlichungen von neuen Untersuchungen auf dem Gebiete der Dispersoidchemie, welche von mir und von meinen Schülern im speziell dazu eingerichteten Laboratorium angestellt worden sind, einen systematischen und geregelten Charakter haben werden 3 ).

Zum Schluß möchte ich der besseren Uebersicht wegen die Arbeiten anführen, welche als Fortsetzungen der Abhandlung ${ }_{n} \mathrm{Zur}$ Lehre von den Zustanden der Materie" dienen:

1) Vgl. meine SchluBbemerkung zu $\$ 29$ in Koll.- Leitschr. 5, 221 (1909).

*) Der erste Teil des Manuskinpts wurde 1907 der Redaktion zugestellt, der Druck desselben aber durch die Uebersetzung verzögert.

s) Dank der Eluwilligung des Herausgebers vorliegender Zeitschritt werden diese Abhandlungen (welche hauptsächlich eine genaue experimentulle Bearbeitung der in meinen friheren Arbeiten nur kurz mitgeteilten oder angedeuteten Daten enthalten) periodisch in den Kolloldchem. Beiheften " unter dem Titel. Dispersoldchemische Mitteilungen aus dein Laboratorian der physikalischen Chemie des Berginstituts der Kaiserin Katharina Il., St. Petersburg " erscheinen [vgl. ebenfalls Grunc!$z$ äge der Dispersoidchemie $119 \ldots 121$ (1911)]. 


\section{Kolloidchemische Beihefte.}

1. Uebersättigung und Unterkühlungals grundlegende Ursachen des dispersen Zustandes der Materie. 1, 331 (1910).

2. Die Theorie der Herstellung und der Stabilitat kolloider Lösungen, I. 1, $396^{\circ}(1910)$ - II. 4, 101 (1912).

3. Zur Systematik des Aggregatzustandes der Materie. 4, 65 (1912).

\section{Kolloid-Zeitschrift.}

4. Ueber einige Oberflacheneigenschaften disperser kristallinischer Stoffe und über die Beziehung dieser Eigenschaften zu dem Dispersitätsgrad. 6, 32 (1910).

5. Zur Geschichte meiner Vektorialitätstheorie der Materie. 7, $\left.256(1910)^{2}\right)$.

6. Theorie der Uebergangserscheinungen zwischen Kolloiden und wahren Lösungen. 8, 24 (1911).

12.125. September 1911.

7) Vgl. ebentalls P. P. von Weimarn, Grundzüge der Dispersoldchemie " (Dresden 1911, Verlag von Theodor Steinkopff).

\section{Erklärung zu den Tafeln.}

Die soeben beschriebenen Versuche mit Propylalkohol und einer gesăttigten wăsserigen Losung von $\mathrm{NaCl}$ sind dennoch weniger lehrrelch 1), als die, welche mir zu meinen Demonstrationen wahrend der Vorlesunger über Kolloidchemie in der Kaiserlichen St. Petersburger Universităt dienen.

Diese Vorlesungsdemonstrationen werden in der voriregenden Abhandlung durch die Tateln I, II und III illustriert; der Inhalt derselben wird vollkommen verstăndlich durçh folgende kurze Erlăuterungen:

Auf Tafel I zeigt Fig. 1 zwei Kristalldrusen von $\mathrm{NaCl}$, welche sich in der Natur bei sehr langsamer Bilding der festen Phase aus groBen Losungsmengen gebildet haben; die fünf ersten Kristalle der Fig. 2 auf Tafel I haben sich gieichfalls in der Natur gebildei (sogen. angeschossenes Sal $z^{*}$ ), die übrigen drei wurden bei der Kristallisalion wăsseriger Losungen von Na Cl unter den gewöhnlichen Bedingungen im Lahoratorium erhalten. Alle Kristalle der Tafel I sind in ca. 1/: naturlicher Große abgebildet.

1) AuBerdem erfordert ihre Ausführung eine bedeutende Fertigkeit in der schnellen Her stelleng mikroskopischer Präparate, da der Propylalkohol sich rasch verflüchtigt und sich die Loslichkeit des Niederschlages vergröbert, wodurch sich seine Körner rascher umkrisiallisieren. 
Fig. 3. $\mathrm{Zu} 15 \mathrm{~cm}$ einer gesättigten wăsserigen Losung von $\mathrm{NaCl}$ wurden $20 \mathrm{ccm}$ Methylalkohol zugefügt; 15 Minuten nach der Mischung beider Flussigketten wurde der Niederschlag auf einen Objektträger übergeführt, nit einem Deckglas bedeckt und photographiert.

Wie aus dem Mikrogramm ersichtlich, besteht der Nlederschlag aus Würfeln (Objektiv 4 b, Okular 18, VergröBerung ca. $300 \mathrm{mal}$ ).

Die Niederschläge von $\mathrm{NaCl}$ in den Tafein II und III sind auf folgende Weise erhalten worden:

Fig. 4. $\mathrm{Zu} 40 \mathrm{ccm}$ Propylalkohol wurden $4 \mathrm{ccm}$ einer gesattigten Lósung von $\mathrm{NaCl}$ in Methylalkohol zugesetzt; nach 30 Minuten wurde der Niederschlag unter denselben Bedingungen photographiert wie im vorhergehenden Fall.

Der Niederschlag besteht aus Würfeln (sehr wenigen) und Wurfel-Oktocdern; die Kanten des Oktoeders sind nicht volistăndig regelrecht ausgebildet ${ }^{1}$ ). Infolge vou Lichtteflexen sind die Wirfel-Oktoeder auf Fig. 4 nicht reliefartig herausgekommen.

Fig. $5.4 \mathrm{ccm}$ einer gesättigten Lösung von $\mathrm{NaCl}$ in Methylalkohol wurden in ein Gemisch von $30 \mathrm{ccm}$ Propylalkohol und $10 \mathrm{ccm}$ Aether eingegossen. Nach 1 Stunde 20 Minuten wurde nach Absitzenlassen der Niederschlag photographiert (dic Herstellung des Präparates und die Vergröberung waren dieselben, wie bei Fig. 3 und 4). Der Niederschlag zeigt Wachstumsform ${ }^{2}$ ).

Fig. 6. $4 \mathrm{ccm}$ einer gesattigten Lösung von $\mathrm{NaCl}$ in Methylalkohol wurden in ein Gemisch von $20 \mathrm{ccm}$ Propylalkohol und $20 \mathrm{ccm}$ Aether gegossen. Nach 3 Stunden 30 Minuten hatte sich die Flussigkeit nocil nicht vollkommen gekJart, doch hatte sich ein bedeutender Teil des Niederschtages auf dem Boden des Probierglases angesammelt. Dieser Niederschlag wurde denn auch photographiert, jedoch bei einer sechsmal starkeren VergröBerung, als die Niederschläge von Fig. $3-5$ (Objektiv $3 \mathrm{~mm}$, Okular 18, Vergrőerung $1800 \mathrm{mal}$ ), da bei eincr 300 maligen VergroBerung der Niederschlag "a morph", feinkornig, erscheint.

Wie aus Fig. 6 ersichtlich, stellen die gröberen Körner Kristaliskelette dar.

Fig. 7. In eine Mischung aus $30 \mathrm{~cm}$ Aether und $10 \mathrm{ccm}$ Propylalkohol wurden $4 \mathrm{ccm}$ einer gesättigten $\mathrm{NaCl}$-Lösung in Methylalkohol gegossen. Man erhält eine milchig trübe Flüssigkeit, welche sich in 24 Stunden nicht vollständig aufhellt, jedoch einen voluminösen Niederschlag von feinkörniger, „, a morphe r “ Struktur bildet.

Fig. 8. In $40 \mathrm{ccm}$ Aether wurden $4 \mathrm{~cm}$ einer gesättigten Lösung von $\mathrm{NaCl}$ in Methylalkohcl gegossen. Die Flïssigkeit ist stark opaleszierend mit der für dispersoide Systeme charakteristischen rotbraunen Färbung bei durchfallendem Licht. Nach 24 Stunden erhält nuan einen ganz getingftlgigen Niederschlag (die Mikrophotographie Ist in Fig. $8^{3}$ ) gegeben), die Flüssigkeit bleibt im übrigen unverandert.

1) Die kristallographische Untersuchung dieses Niederschlages wird in Berg-Institut ansgefübrt werden.

3) Die Obetfläche det Wachstunsform ist bei gleichem Volumen gröfier als die des rollkantigen Kristalle.

8) Es ist nôtig, zu berrerken, dab bei diesen Niederschlägen (Fig. 7 ind Pig. 8) sich leicht sekurdūte ,Wabenstruktures:" usw. bilden. 
Wenn man eine geringere Menge als $4 \mathrm{ccm}$ der gesăttigten $\mathrm{NaCl}$-Lösung in Methylalkohol in stark abgekühlten Aether (z. B. 800) eingieBt, so erhulk man dispersoide Losungen fast bis zun molekularen Dispersitätsgrade.

Man muB im Auge behalten, daß die Löslichkeit der festen Phasc von $\mathrm{NaCl}$ in den angefihten Gemischen sich progressiv verringert, die Schnelligkeit threr Bildung dagegen wàchst.

Die hier zu beobachtenden Bezlehungen sind dem Wesen nach diesclben wic bei $\mathrm{BaSO}_{4}$, welche bereits in meinen fruheren Arbeiten beschrieben sind.

Als Ergänzung $7 u$ den Abbildungen der $\mathrm{BaSO}_{4}$-Niederschläge bringe ich hier Abbildungen von naturlichen Kristallen von schwefelsaurem Harium (Fig. 9)

Es ist wichtig, zu bemerken, daß man nicht nur $\mathrm{NaCl}$, sondern auch alle Salze der Alkalimetalle ( $\mathrm{Na}, \mathrm{K}, \mathrm{Li}, \mathrm{Rb}, \mathrm{Cs}, \mathrm{NH}_{4}$ ) von den Typen: $\mathrm{RCl}, \mathrm{RBr}$, RJ, RCN, RCNS, $\mathrm{R}_{2} \mathrm{CO}_{3}, \mathrm{R}_{2} \mathrm{SO}_{4}, \mathrm{RNO}_{3}$ auf dieselbe Weise in den verschiedenen Dispersitătsgraden erhalten kann (P.P. von WeImarn und A. L. Stein! und es besteht kein Zweifelan der Allgemeingiultigkeit der angegebenen Methode (siehe meine frïheren Arbeiten) fiir beliebige Körper, 